\title{
Signatures of nonlinear mode interactions in the pulsating hot B subdwarf star KIC 10139564
}

\author{
W. Zong ${ }^{1,2}$, S. Charpinet ${ }^{1,2}$, and G. Vauclair ${ }^{1,2}$ \\ 1 Université de Toulouse, UPS-OMP, IRAP 31400 Toulouse, France \\ e-mail: [weikai .zong; stephane.charpinet; gerard.vauclair]@irap.omp.eu \\ 2 CNRS, IRAP, 14 avenue Edouard Belin, 31400 Toulouse, France
}

Received 16 June 2016 / Accepted 18 July 2016

\begin{abstract}
Context. The unprecedented photometric quality and time coverage offered by the Kepler spacecraft has opened up new opportunities to search for signatures of nonlinear effects that affect oscillation modes in pulsating stars.

Aims. The data accumulated on the pulsating hot B subdwarf KIC 10139564 are used to explore in detail the stability of its oscillation modes, focusing in particular on evidences of nonlinear behaviors.

Methods. We analyzed 38 months of contiguous short-cadence data, concentrating on mode multiplets induced by the star rotation and on frequencies forming linear combinations that show intriguing behaviors during the course of the observations.

Results. We find clear signatures that point toward nonlinear effects predicted by resonant mode coupling mechanisms. These couplings can induce various mode behaviors for the components of multiplets and for frequencies related by linear relationships. We find that a triplet at $5760 \mu \mathrm{Hz}$, a quintuplet at $5287 \mu \mathrm{Hz}$ and a $(\ell>2)$ multiplet at $5412 \mu \mathrm{Hz}$, all induced by rotation, show clear frequency and amplitude modulations which are typical of the so-called intermediate regime of a resonance between the components. One triplet at $316 \mu \mathrm{Hz}$ and a doublet at $394 \mu \mathrm{Hz}$ show modulated amplitude and constant frequency which can be associated with a narrow transitory regime of the resonance. Another triplet at $519 \mu \mathrm{Hz}$ appears to be in a frequency-locked regime where both frequency and amplitude are constant. Additionally, three linear combinations of frequencies near $6076 \mu \mathrm{Hz}$ also show amplitude and frequency modulations, which are likely related to a three-mode direct resonance of the type $v_{0} \sim v_{1}+v_{2}$.

Conclusions. The identified frequency and amplitude modulations are the first clear-cut signatures of nonlinear resonant couplings occurring in pulsating hot B subdwarf stars. However, the observed behaviors suggest that the resonances occurring in these stars usually follow more complicated patterns than the simple predictions from current nonlinear theoretical frameworks. These results should therefore motivate further work to develop the theory of nonlinear stellar pulsations, considering that stars such as KIC 10139564 now offer remarkable testbeds to do so.
\end{abstract}

Key words. techniques: photometric - stars: variables: general - stars: individual: KIC 10139564

\section{Introduction}

Hot B subdwarf (sdB) stars are helium core burning objects that populate the so-called extreme horizontal branch (EHB). They are expected to have a mass around $0.47 M_{\odot}$ and are characterized by a very thin hydrogen-rich residual envelope containing at most $\sim 0.02 M_{\odot}$. For this reason, they remain hot and compact throughout all their helium core burning evolution, with effective temperatures, $T_{\text {eff }}$, and surface gravities, $\log g$, ranging from $22000 \mathrm{~K}$ to $40000 \mathrm{~K}$ and from 5.2 to 6.2, respectively (Heber 2009; Fontaine et al. 2012).

The presence of pulsations in some sdB stars make them good candidates for probing their interior with the technique of asteroseismology. A first group of nonradial sdB pulsators with periods of a few minutes was theoretically predicted by Charpinet et al. (1996) and effectively discovered by Kilkenny et al. (1997). These pulsators, now referred to as the V361 Hya stars, show low-order, low-degree pressure ( $p$-)modes that are driven by a $\kappa$-mechanism induced by the partial ionization of iron-group elements occurring in the "Z-bump" region and powered-up by radiative levitation (Charpinet et al. 1996, 1997). Long period oscillations of $\sim 1-4 \mathrm{~h}$ were later discovered by Green et al. (2003), forming another group of
sdB pulsators known as the V1093 Her stars. The latter show mid-order gravity $(g$-)modes driven by the same mechanism (Fontaine et al. 2003). Hybrid pulsators that show both $p$ - and $g$-mode oscillations simultaneously have also been reported (e.g., Schuh et al. 2006). Tight seismic constraints have indeed been obtained from the measured frequencies using both types of sdB pulsators, in particular based on high-quality photometric data gathered from spaceborne telescopes (e.g., Charpinet et al. 2011; van Grootel et al. 2010). However, the reason behind the apparent variability of some oscillation modes in sdB stars, already noticed from repeated ground based campaigns (e.g., Kilkenny et al. 2007), has remained poorly understood.

The temporal variation of oscillation modes in pulsating sdB stars is beyond the scope of the standard linear nonradial stellar oscillation theory in which eigenmodes have a stable frequency and amplitude (Unno et al. 1989). These behaviors must be studied within a nonlinear framework to interpret the modulations. In particular nonlinear resonant mode coupling effects are expected to affect some oscillation modes, as noted, for example, in the helium dominated atmosphere white dwarf variable (DBV) star GD 358 (Goupil et al. 1998). Different types of resonant coupling have been investigated within the framework of the amplitude equation (AE) formalism since the 1980's 
(e.g., Buchler \& Goupil 1984), among them the $v_{0} \sim v_{1}+v_{2}$ resonance (Dziembowski 1982; Moskalik 1985) and the 2:1 resonance in Cepheid stars (Buchler \& Kovacs 1986). The AE formalism was then extended to nonadiabatic nonradial pulsations in Eulerian and Lagrangian formulations by Goupil \& Buchler (1994) and van Hoolst (1994), respectively. A theoretical exploration of specific cases of nonradial resonances was developed in Buchler et al. (1995, 1997), including notably the resonance occurring in a mode triplet that is caused by slow stellar rotation and which satisfies the relationship $v_{+}+v_{-} \sim 2 v_{0}$, where $v_{0}$ is the frequency of the central $m=0$ component. However, these theoretical developments based on AEs have since considerably slowed down, in part due to the lack of clear observational data to rely on.

The launch of instruments for ultra high precision photometry from space has changed the situation, making it now possible to capture amplitude and/or frequency modulations occurring on timescales of months or even years that were difficult to identify from ground-based observatories. It is however from ground based data that Vauclair et al. (2011) proposed for the first time that resonant couplings within triplets could explain the long-term variations, both in amplitude and frequency, seen in several oscillation modes monitored in the GW Virginis pulsator PG $0122+200$, through successive campaigns.

The observation of a multitude of pulsating stars, including sdB and white dwarf stars, by the Kepler spacecraft has open up new opportunities to identify and characterize the mechanisms that could modulate the oscillation modes. Kepler monitored a $105 \mathrm{deg}^{2}$ field in the Cygnus-Lyrae region for around four years without interruption, thus obtaining unprecedented high quality photometric data for asteroseismology (Gilliland et al. 2010). These uninterrupted data are particularly suited for searching long-term temporal amplitude and frequency modulations. In the context of white dwarf pulsators, for instance, Zong et al. (2016, hereafter Z16) found that the DBV star KIC 08626021 shows clear signatures of nonlinear effects attributed to resonant mode couplings. In this star, three rotational multiplets show various types of behaviors that can be related to different regimes of the nonlinear resonant mode coupling mechanism. In particular some amplitude and frequency modulation timescales are found to be consistent with theoretical expectations. This finding suggests that the variations of some oscillation modes in sdB stars may also be related to nonlinear resonance effects. It is in this context that we decided to search clues of similar nonlinear phenomena involving mode interactions in pulsating sdB stars.

Eighteen sdB pulsators have been monitored with Kepler (see Østensen et al. 2014 and references therein). In this paper, we focus on one of them, the star KIC 10139564, which was discovered in quarter Q2.1 and then continuously observed from Q5.1 to Q17.2. A preliminary analysis based on one month of short cadence data originally showed that KIC 10139564 is a V361-Hya type (rapid, $p$-mode) sdB pulsator featuring also a low-amplitude $g$-mode oscillation (Kawaler et al. 2010). With extended data, Baran et al. (2012) detected up to 57 periodicities including several multiplets attributed to the rotation of the star. These multiplets are characterized by common frequency spacings, both for the $p$ - and $g$-modes, indicating that KIC 10139564 has a rotation period of $25.6 \pm 1.8 \mathrm{~d}$. These authors did not find any radial-velocity variations from their dedicated spectroscopy and derived the atmospheric parameter values $T_{\text {eff }}=31859 \mathrm{~K}$ and $\log g=5.673$ for this star. An interesting finding concerning KIC 10139564 is that two of the identified multiplets may have degrees $\ell$ greater than two, a possibility further investigated by Baran \& Østensen (2013). The detection of several multiplets in this star continuously monitored for more than three years makes it a target of choice for studying eventual nonlinear resonant mode couplings in sdB stars.

In this study, we show that several multiplets in KIC 10139564 have indeed amplitude and frequency modulations suggesting nonlinear resonant mode couplings, which constitutes the first clear-cut case reported for $\mathrm{sdB}$ pulsators, so far. In Sect. 2, we present the thorough analysis of the frequency content of the Kepler photometry available on KIC 10139564, including our analysis of the frequency and amplitude modulations identified in several multiplets and linear combination frequencies. In Sect. 3, we recall some theoretical background related to nonlinear resonant mode couplings, focusing mainly on two types of resonances. The interpretation of the observed modulations which may relate to nonlinear resonant mode couplings is discussed in Sect. 4. The summary and conclusion are then given in Sect. 5 .

\section{The frequency content of KIC 10139564 revisited}

\subsection{The Kepler photometry}

The pulsating sdB star KIC 10139564 was observed by Kepler in short-cadence (hereafter SC) mode during quarter Q2.1 and from Q5.1 to Q17.2 (i.e., until the spacecraft finally lost its second inertia reaction wheel and stopped its operations). Results based on parts of these data have already been published in the literature (e.g., Baran et al. 2012; Baran \& Østensen 2013). we obtained the light curves through the Kepler Asteroseismic Science Consortium (KASC) ${ }^{1}$. These data were processed through the standard Kepler Science Processing Pipeline (Jenkins et al. 2010). For our purposes, we do not further consider the "short" (one month) light curve of Q2.1 which is well disconnected from the main campaign and would introduce a large and detrimental gap for our upcoming analysis. This leaves us with a nearly contiguous 38-month light curve starting from BJD 2455276.5 and ending on BJD 2456424 (which spans 1147.5 days), with a duty circle of $\sim 89 \%$.

The full light curve was constructed from each quarter "corrected" light curves, which most notably include a correction of the amplitudes taking into account contamination by nearby objects (this correction estimates that $\sim 83.2 \%$ of the light comes from KIC 10139564). Each quarter light curve was individually detrended to correct for residual drifts by performing a sixthorder polynomial fit. Then, data points that differ significantly from the local standard deviation of the light curve were removed by applying a running $3 \sigma$ clipping filter. Note that the latter operation decreases slightly the overall noise level in Fourier space, but has no incidence on the measured frequencies.

The fully assembled light curve of KIC 10139564 is shown in the top panel of Fig. 1 while the bottom panel expands a 0.8-day portion of the data. Low-amplitude multi-periodic oscillations dominated by periodicities of a few minutes are clearly visible. Their presence is confirmed in the corresponding Lomb-Scargle periodogram (LSP, Fig. 2; Scargle 1982). The LSP shows two distinct regions with significant signal corresponding to $p$-modes at high frequencies and $g$-modes at low frequencies. This identifies KIC 10139564 as a hybrid pulsating sdB star (Schuh et al. 2006) whose oscillations are however largely dominated by $p$-modes. The formal frequency resolution achieved with these data is $\sim 0.010 \mu \mathrm{Hz}$.

http://astro.phys.au.dk/KASC 


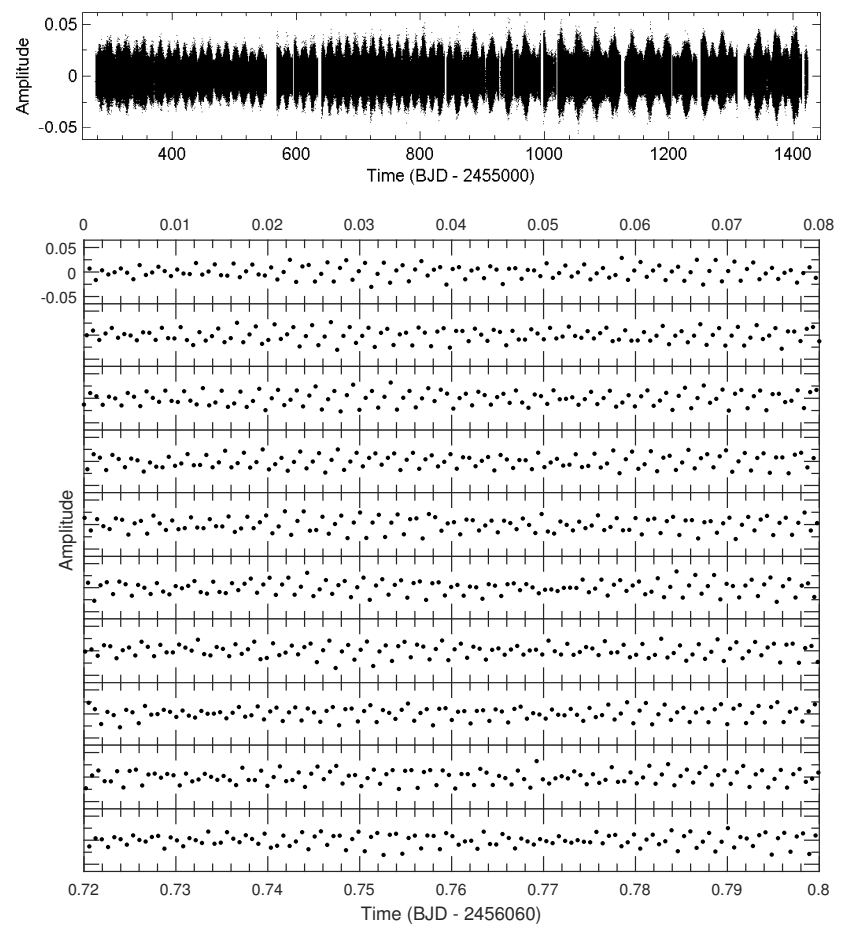

Fig. 1. Top panel: condensed representation of the full Kepler light curve of KIC 10139564 (Amplitude as the residual relative to the mean brightness intensity of the star vs time in Barycentric Julian Date) covering from Q5.1 to Q17.2 ( 1147.5 days). Bottom panel: close-up view showing 0.8 days of the Kepler light curve by slices of 0.08 days. At this scale the oscillations are clearly apparent.

\subsection{Frequency extraction}

A dedicated software, FELIX (Frequency Extraction for LIghtcurve eXploitation) developed by one of us (S.C.), was used to first extract the frequency content of KIC 10139564 down to a chosen detection threshold. The latter was established following the same method as in Z16 (see their Sect. 2.2), leading also in the present case to a conservative $5.6 \sigma$ criterion (in practice, we searched down to $\sim 5 \sigma$ if a frequency is suspected to be part of a multiplet; see below).

The extraction method is a standard prewhithening and nonlinear least square fitting technique (Deeming 1975), which works with no difficulty in the present case. The code FELIX greatly accelerates and eases the application of this procedure, especially for treating very long time-series obtained from space such as CoRoT and Kepler (Charpinet et al. 2010, 2011).

We provide in Table A.1 (see Appendix) a list of all the extracted frequencies with their fitted attributes (frequency in $\mu \mathrm{Hz}$, period in second, amplitude in percent of the mean brightness, phase relative to a reference time $t_{0}$, and signal-to-noise ratio) and their respective error estimates $\left(\sigma_{\mathrm{f}}, \sigma_{\mathrm{P}}, \sigma_{\mathrm{A}}\right.$, and $\left.\sigma_{\mathrm{Ph}}\right)$. For convenience, because in this study we focus on a particular subset of the observed frequencies, we repeat some of these information in Table 1 for the relevant modes. The "Id." column in both tables uniquely identify a detected frequency with the number indicating the rank by order of decreasing amplitude.

We have detected 60 clear independent frequencies that comes out well above the $5.6 \sigma$ detection threshold (Table A.1), of which 29 frequencies consist of three triplets, one doublet, one quintuplet and two incomplete multiplets with $\ell>2$ (Table 1). We also detect another three frequencies that appear as significant but are linked to other frequencies through linear combinations. Five additional "forests" of frequencies, each containing many close peaks in a very narrow frequency range, are detected in the $5400-6400 \mu \mathrm{Hz}$ region. We also prewhitened 14 frequencies whose amplitudes are above $5.0 \sigma$ but below $5.6 \sigma$ which, we suspect, are real pulsations. Our well-secured extracted frequencies agree well with the independent analysis of Baran \& Østensen (2013), but we detect a few more lowamplitude frequencies because the data that we consider here cover about one more year. We do not investigate further these "forests" of frequencies (G1-G5, see Table A.1) that show very complicated structures. These were discussed in Baran et al. (2012). We point out that our extracted frequencies may differ in amplitude compared with the work of Baran \& Østensen (2013) because some of these frequencies have variable amplitudes.

\subsection{Error estimates on frequencies and amplitudes}

Before proceeding further in our analysis, we briefly discuss our quantitative evaluation of the uncertainties associated with the measured frequencies and amplitudes given in Tables 1 and A.1. The reliability of these error estimates is particularly important when it comes to discuss amplitude and frequency variations with time, in particular to assess if these are significant or not.

With FELIX, errors are estimated following the formalism proposed by Montgomery \& Odonoghue (1999), with the particularity, however, that $\sigma_{\mathrm{A}}$, the error on the amplitude of a mode, is measured directly in the Lomb-Scargle periodogram. A window around each frequency is chosen and the median value of the amplitudes in that frequency range defines $\sigma_{\mathrm{A}}$. The relations given in Montgomery \& Odonoghue (1999) are then used to compute the other errors, in particular $\sigma_{\mathrm{f}}$, the error on the measured frequency. In order to test that this procedure is correct and does not largely under or overestimate the true errors, we conduct two Monte Carlo experiments.

We first construct an artificial light curve covering about 200 days (similar to the time baseline of each light curve pieces considered in the next subsection) with the same SC-mode sampling provided by Kepler in which we add white random gaussian noise. We further inject in this light curve 1000 sinusoidal signals with the same amplitude $(S / N \sim 160)$ but of frequency increasing by steps of $\sim 8.2 \mu \mathrm{Hz}$ per signal. In practice, a random frequency shift of a few tenth $\mu \mathrm{Hz}$ is performed on each injected frequency in order to reduce the number of harmonics and linear combinations. The generated time series is then analyzed with our code FELIX that extracts and measures each signal and evaluates the uncertainties associated to the measured frequencies and amplitudes $\left(\sigma_{\mathrm{A}}\right.$ and $\left.\sigma_{\mathrm{f}}\right)$. Since the true values of these quantities are perfectly known from the signals we injected, the real distribution of the deviations between measured (prewhitenned) values and true values can be evaluated. For that purpose, we define the frequency and amplitude deviations normalized by their $1 \sigma$ errors (as estimated with the code FELIX from the procedure described above), $\Delta f=\left(f_{\text {pre }}-f_{\text {inj }}\right) / \sigma_{\mathrm{f}}$ and $\Delta A=\left(A_{\text {pre }}-A_{\text {inj }}\right) / \sigma_{\mathrm{A}}$, where the subscripts indicate the prewhitened value and the injected one, respectively. A variant of this test is also performed by again injecting 1000 sinusoidal signals, but this time with random amplitudes (instead of constant ones) chosen in the $S / N \in(5,60)$ range. This second test allows us to check also the reliability of our error estimates as a function of amplitude, considering that $\sigma_{\mathrm{f}}$ in particular depends on the mode $\mathrm{S} / \mathrm{N}$ ( $\sigma_{\mathrm{f}}$ increases when $\mathrm{S} / \mathrm{N}$ decreases).

Figure 3 shows the results obtained in both cases. The 2D distributions of the frequency and amplitude deviations are well confined within $3 \sigma$. Moreover, the associated 


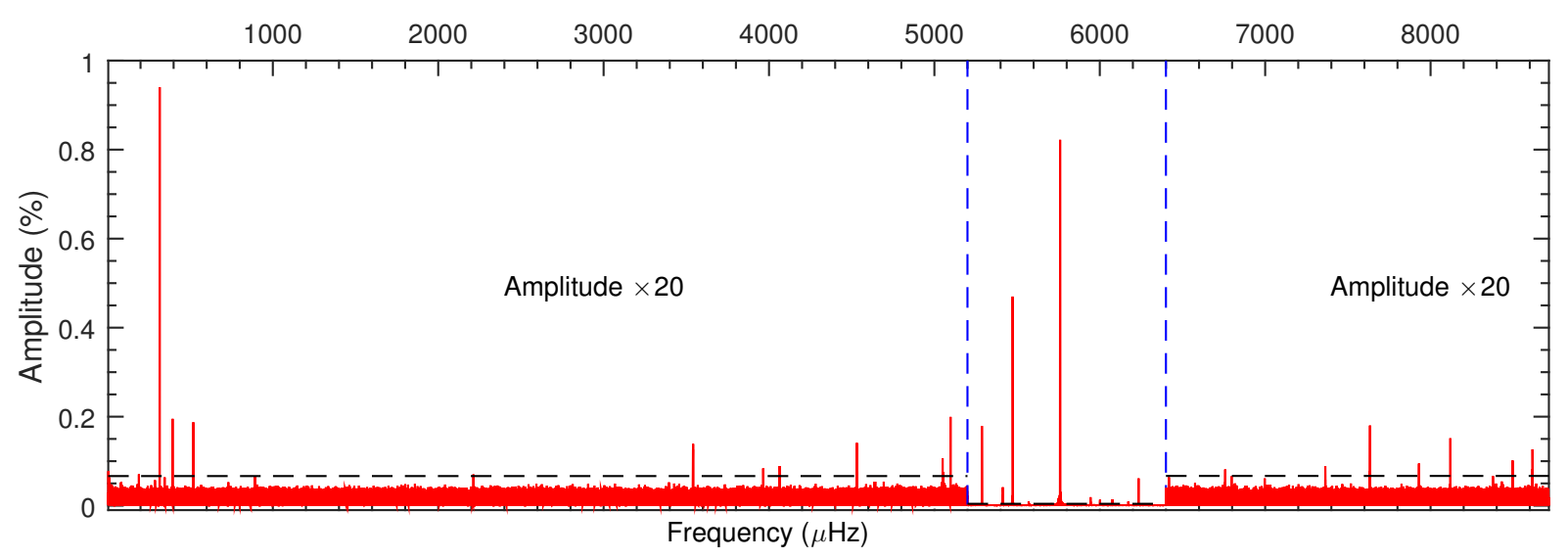

Fig. 2. Lomb-Scargle periodogram (LSP; amplitude in \% of the mean brightness vs frequency in $\mu \mathrm{Hz}$ ) of the Kepler light curve for KIC 10139564 . The represented range, up to the Nyquist frequency, covers the long-period $g$-mode and the short-period $p$-mode frequency domains. The region between the two dashed vertical lines at 5200 and $6400 \mu \mathrm{Hz}$ is where peaks have the largest amplitudes. However, weaker peaks outside of this particular region are present and are made visible by scaling up amplitudes by a factor of 20 . The dashed horizontal line represents the $5.6 \sigma$ detection threshold (see text). Some well-known Kepler instrumental artefacts are present, but can easily be recognized.

Table 1. List of frequencies detected in KIC 10139564 on which we focus our analysis.

\begin{tabular}{|c|c|c|c|c|c|c|c|c|c|}
\hline Id. & $\begin{array}{c}\text { Frequency } \\
(\mu \mathrm{Hz})\end{array}$ & $\begin{array}{c}\sigma_{\mathrm{f}} \\
(\mu \mathrm{Hz})\end{array}$ & $\begin{array}{l}\text { Period } \\
(\mathrm{s})\end{array}$ & $\begin{array}{l}\sigma_{\mathrm{P}} \\
(\mathrm{s})\end{array}$ & $\begin{array}{l}\text { Amplitude } \\
(\%)\end{array}$ & $\begin{array}{l}\sigma_{\mathrm{A}} \\
(\%)\end{array}$ & Phase & $\sigma_{\mathrm{Ph}}$ & $S / N \dagger$ Comment \\
\hline
\end{tabular}

\section{Multiplet frequencies:}

$\begin{array}{lll}f_{39} & 315.579243 & 0.000566 \\ f_{21} & 315.820996 & 0.000219 \\ f_{11} & 316.066440 & 0.000070 \\ & & \\ f_{27} & 394.027385 & 0.000342 \\ f_{32} & 394.289823 & 0.000397 \\ & & \\ f_{34} & 518.900359 & 0.000437 \\ f_{28} & 519.151796 & 0.000352 \\ f_{31} & 519.402391 & 0.000367\end{array}$

$f_{31} 519.402391$

$f_{08} \quad 5286.149823$

$f_{10} 5286.561766$

$f_{07} 5286.976232$

$f_{05} \quad 5287.391879$

$\begin{array}{ll}f_{06} & 5287.805883\end{array}$

$f_{22} \quad 5410.701146$

$\begin{array}{ll}f_{67} & 5411.143448\end{array}$

$\begin{array}{ll}f_{13} & 5411.597301\end{array}$

$f_{15} \quad 5412.516444$

$\begin{array}{ll}f_{12} & 5413.389096\end{array}$

$\begin{array}{ll}f_{19} & 5413.814342\end{array}$

$f_{01} \quad 5760.167840$

$\begin{array}{ll}f_{03} & 5760.586965\end{array}$

$f_{02} \quad 5761.008652$

\subsection{3 \\ 0.000060 \\ 0.000038 \\ 0.000019 \\ 0.000029}

0.000234

0.000958

0.000136

0.000185

0.000084

0.000222

0.000005
0.000008
0.000007
$3168.776214 \quad 0.005687$

3166.3505990 .002193

0.005851

0.015155

3163.8917440 .000702

0.047276

$\begin{array}{lll}0.000596 & 0.2492 & 0.0516\end{array}$

$\begin{array}{llll}0.000596 & 0.6107 & 0.0199\end{array}$

$\begin{array}{llllll}2537.894669 & 0.002202 & 0.009667 & 0.000594 & 0.2589 & 0.0312\end{array}$

$\begin{array}{lllllll}2536.205455 & 0.002555 & 0.008323 & 0.000594 & 0.5123 & 0.0363\end{array}$

$\begin{array}{lllllll}1927.152262 & 0.001624 & 0.007526 & 0.000592 & 0.6648 & 0.0401\end{array}$

$\begin{array}{lllllll}1926.218898 & 0.001305 & 0.009351 & 0.000592 & 0.9059 & 0.0323\end{array}$

$\begin{array}{lllllll}1925.289559 & 0.001360 & 0.008964 & 0.000592 & 0.5369 & 0.0337\end{array}$

$\begin{array}{lllllll}189.173601 & 0.000002 & 0.064784 & 0.000614 & 0.6712 & 0.0047\end{array}$

$\begin{array}{lllllll}189.158861 & 0.000002 & 0.057105 & 0.000614 & 0.4356 & 0.0053\end{array}$

$\begin{array}{lllllll}189.144032 & 0.000001 & 0.088857 & 0.000614 & 0.1202 & 0.0034\end{array}$

$\begin{array}{lllllll}189.129163 & 0.000001 & 0.179339 & 0.000615 & 0.3374 & 0.0017\end{array}$

$\begin{array}{lllllll}189.114355 & 0.000001 & 0.119329 & 0.000615 & 0.7941 & 0.0025\end{array}$

$\begin{array}{lllllll}184.818931 & 0.000008 & 0.014871 & 0.000627 & 0.9524 & 0.0203\end{array}$

$\begin{array}{lllllll}184.803824 & 0.000033 & 0.003637 & 0.000627 & 0.4591 & 0.0830\end{array}$

$\begin{array}{lllllll}184.788325 & 0.000005 & 0.025636 & 0.000627 & 0.6770 & 0.0118\end{array}$

$\begin{array}{lllllll}184.756944 & 0.000006 & 0.018812 & 0.000627 & 0.8925 & 0.0160\end{array}$

$\begin{array}{lllllll}184.727161 & 0.000003 & 0.041339 & 0.000627 & 0.4037 & 0.0073\end{array}$

$\begin{array}{lllllll}184.712651 & 0.000008 & 0.015718 & 0.000627 & 0.7225 & 0.0192\end{array}$

$\begin{array}{lllllll}173.606052 & \ldots & 0.825132 & 0.000761 & 0.0744 & 0.0004\end{array}$

$173.593421 \quad \ldots$

$173.580715 \quad \cdots$

$\begin{array}{llll}0.825132 & 0.000761 & 0.0744 & 0.0004 \\ 0.554646 & 0.000761 & 0.6388 & 0.0005\end{array}$

$\begin{array}{llll}0.567034 & 0.000761 & 0.5845 & 0.0005\end{array}$

$9.8 T_{2,-1}$

$25.4 T_{2,0}$

$79.3 T_{2,+1}$

$16.3 D_{1,0}$

$14.0 D_{1,+1}$

$12.7 T_{3,-1}$

$15.8 T_{3,0}$

$15.2 T_{3,+1}$

$105.4 Q_{1,-2}$

$92.9 Q_{1,-1}$

$144.6 Q_{1,0}$

$291.8 Q_{1,+1}$

$194.2 Q_{1,+2}$

$23.7 M_{1,0}$

$5.8 M_{1,0}$

$40.9 M_{1,0}$

$30.0 M_{1,0}$

$65.9 M_{1,0}$

$25.1 M_{1,0}$

$1084.9 T_{1,-1}$

$729.3 T_{1,0}$

$745.5 T_{1,+1}$

Linear combination frequencies $C_{1}$ :

\begin{tabular}{llllllllllll}
$f_{23}$ & 6076.234996 & 0.000252 & 164.575597 & 0.000007 & 0.014360 & 0.000650 & 0.7906 & 0.0210 & 22.1 & $f_{11}+f_{01}$ \\
$f_{35}$ & 6076.408232 & 0.000510 & 164.570905 & 0.000014 & 0.007091 & 0.000650 & 0.7821 & 0.0426 & 10.9 & $f_{21}+f_{03}$ \\
$f_{74}$ & 6076.650684 & 0.001120 & 164.564338 & 0.000030 & 0.003225 & 0.000650 & 0.5520 & 0.0937 & 5.0 & $f_{11}+f_{03}$ \\
\hline
\end{tabular}

Notes. ${ }^{(\dagger)}$ The first subscript is the identity of the multiplet and the second one indicates the value of $m$. The $m$-values for the $\ell>2$ multiplet $M_{1}$ are not provided, as the degree $\ell$ is not known. 

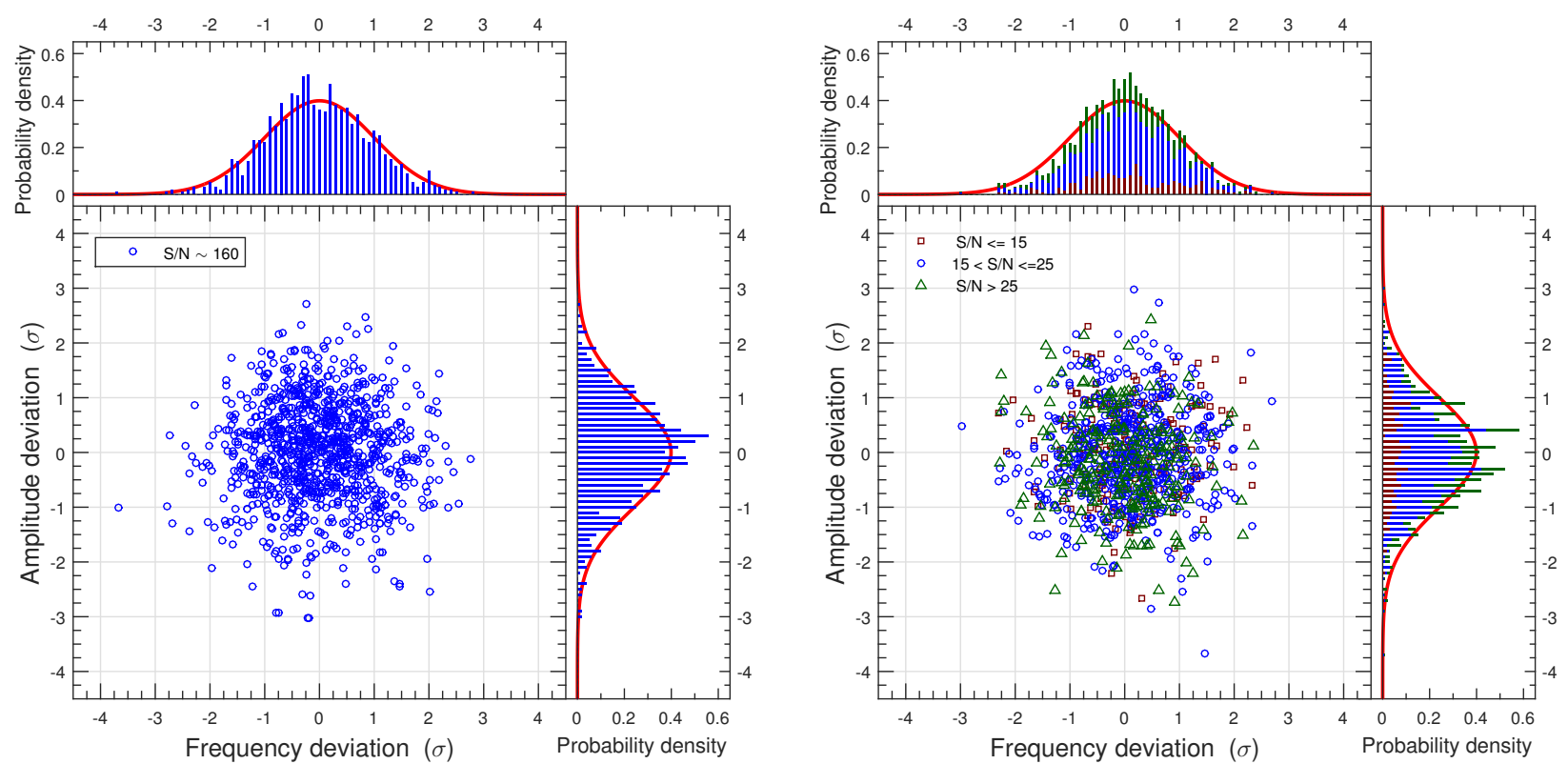

Fig. 3. Left panel: 2D distribution of the frequency and amplitude deviations between the prewhitened and the injected values for 1000 artificial modes of constant amplitude. $\mathrm{S} / \mathrm{N}$ denotes the signal-to-noise ratio of the injected signals and the deviations have been normalized by the $1 \sigma$ error, $\sigma_{\mathrm{A}}$ and $\sigma_{\mathrm{f}}$, derived from the prewhitening procedure implemented in the code FELIX. The 2D distribution is also projected into 1D histograms (frequency and amplitude) to be compared with the Normal Distribution, $\mathcal{N}(0,1)$ plotted as a red solid curve. Right panel: same as above but for 1000 modes with random amplitudes. The injected modes are divided into three groups of $\mathrm{S} / \mathrm{N}$ in the ranges $[5,15],(15,25]$, and $(25,60]$, respectively (represented by three different colors and symbols).

1D histograms show that for both quantities, the measured deviations closely follow the Normal Distribution, $\mathcal{N}(0,1)$, plotted as a red solid curve. Only a few data points fall outside the $[-3 \sigma,+3 \sigma]$ range (within which $99.73 \%$ of the measurements should be for the normal distribution, $\mathcal{N}(0,1))$. This is the behavior we expect for an accurate determination of the error estimates, $\sigma_{\mathrm{A}}$ and $\sigma_{\mathrm{f}}$, with the code FELIX. Hence, these tests demonstrate that error values derived in our frequency analysis are robust.

\subsection{Amplitude and frequency modulations}

From now on, we concentrate our discussion on the six multiplets, which include three triplets $T_{1}, T_{2}$ and $T_{3}$, one doublet $D_{1}$, one quintuplet $Q_{1}$ and a likely $\ell=4$ multiplet $M_{1}$ (see again Table A.1). Interestingly, three of these multiplets $\left(T_{2}, D_{1}\right.$, and $\left.T_{3}\right)$ involve $g$-modes, while the others $\left(Q_{1}, M_{1}\right.$, and $\left.T_{1}\right)$ are $p$-modes. We also examine three linear combination frequencies $\left(C_{1}\right)$. The fine structures of the multiplets are shown in the left top panels of Figs. 4, 6, 7 and 9-11. The average frequency spacing between the components of these well-defined, nearly symmetric multiplets is $\sim 0.25 \mu \mathrm{Hz}$ for the $g$-modes and $0.423 \mu \mathrm{Hz}$ for the $p$-modes, thus suggesting that the $g$-modes are dipoles $(\ell=1)$ in a star rotating rigidly with a period of $\sim 26$ days.

In order to investigate the time variability of these oscillation modes and their relationships, we used our software FELIX to compute sliding Lomb-Scargle periodograms (sLSP) of the data set. This method constructs time-frequency diagrams by filtering in only parts of the data set as a function of time. We chose a filter window of 200-day width moved along the entire light curve by time steps of 20 days. This ensures a good compromise, for our purposes, between the frequency resolution (to resolve close structures of peaks in each LSP), time resolution, and signalto-noise. The sLSP offers an overall view of the amplitude and frequency variations that may occur for a given mode (see, e.g., the middle left panel of Fig. 4). As a complementary (and more precise) technique, we also extracted the frequencies (through prewhitening and nonlinear least square fitting) in various parts of the light curve. The 38-month light curve of KIC 10139564 was divided into 32 time intervals, each containing nine months of Kepler data (for the purposes of precision in the measurements) except for the last three intervals at the end of the observations. This second approach provides a measure of the (averaged) frequencies and amplitudes at a given time, along with the associated errors (see, e.g., the right and bottom left panels of Fig. 4).

Figure 4 shows the amplitude and frequency modulations for the three components forming the triplet $T_{1}$ near $5760 \mu \mathrm{Hz}$. As mentioned already, the top-left panel shows the triplet as revealed by the full data set with components nearly equally spaced in frequency. We note, however, that this spacing is not strictly symmetric, with a difference (or "frequency mismatch") of $0.0026 \mu \mathrm{Hz}$. Frequency variations with time are illustrated by the sLSP diagram in the middle-left panel where the color scale represents the amplitude of the modes. An expanded view centered on the average frequency of each component is then provided in the bottom-left panel while the amplitude behavior with time for each component is shown in the right panel. The latter two are obtained from prewhitening parts of the light curve as described above.

From the sLSP diagram, we find that both the amplitudes and frequencies have varied during the Kepler observations. These variations are more clearly seen in the bottom-left and right panels. The side components both show suggestions of a quasiperiodic modulation in frequency and evolve in antiphase. We also note a long timescale trend as the frequencies of the two side components gradually approach toward each other, as well as toward the central component. In order to filter out these trends, we applied a parabolic fit to each component, leaving the remaining 

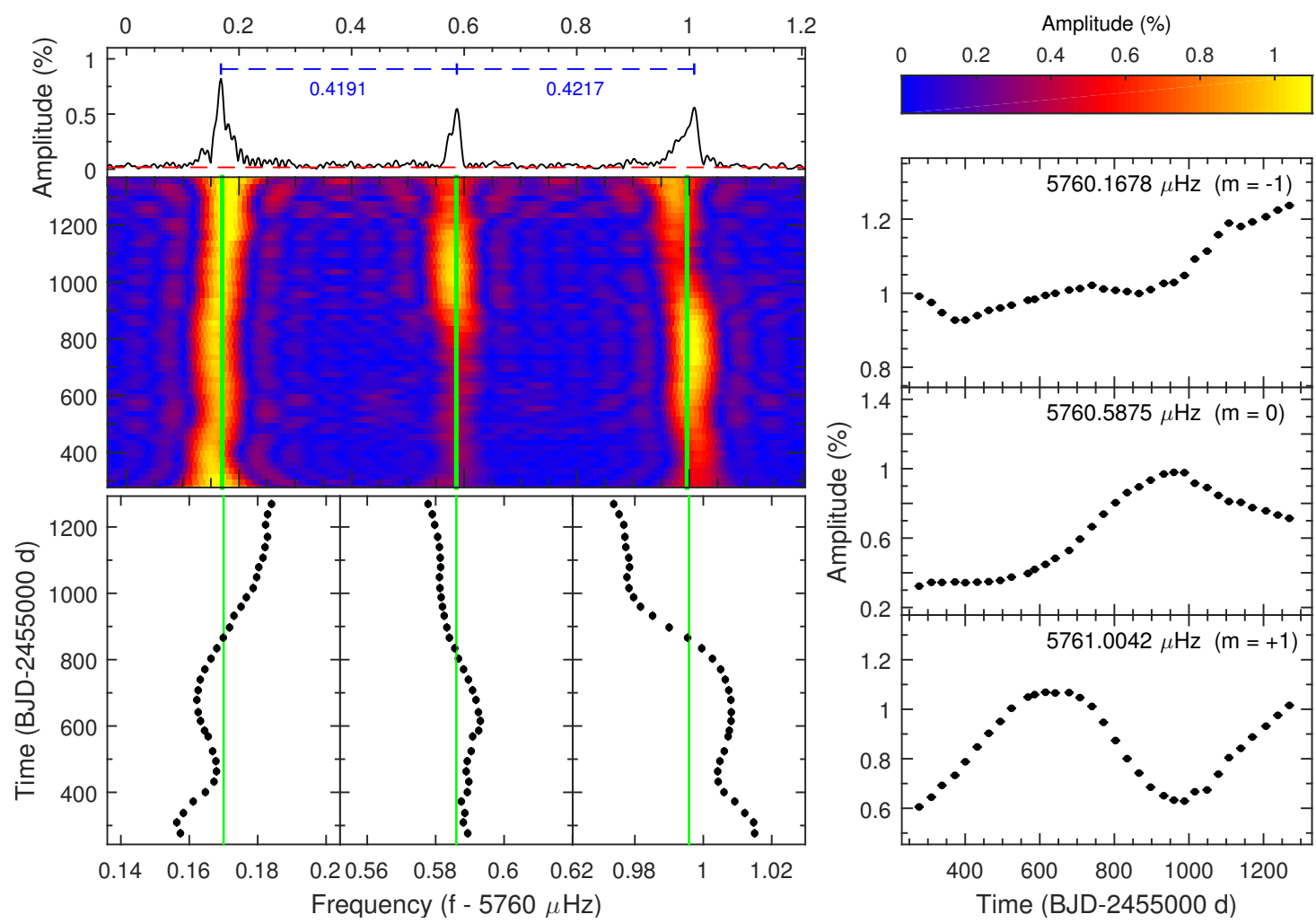

Fig. 4. Frequency and amplitude modulations in the $T_{1} p$-mode triplet near $5760 \mu \mathrm{Hz}$. Top-left panel presents the fine structures of the well defined triplet with near symmetric frequency spacings. The dashed horizontal line in red represents the $5.6 \mathrm{\sigma}$ detection threshold. Middle-left panel shows the sliding Lomb-Scargle periodogram (sLSP giving the amplitude in $\%$ as a function of frequency in $\mu \mathrm{Hz}$ and time in days) of the triplet as a whole. Bottom-left panel shows expanded views around the average frequency (the solid vertical lines, also in the middle left panel) of each component, obtained from prewhitening subsets of the data, thus measuring precisely the frequencies, as a function of time. Right panel provides the measured amplitudes as a function of time obtained for each subset of data (see text for details). Note that the errors for each measurement is smaller than the symbol itself.

signature of the quasi-periodic modulation of the frequencies (see Fig. 5). In the process, we find that the two side components had frequencies about $0.06 \mu \mathrm{Hz}$ closer to each other at the end of the run compared to the beginning of the observations. Figure 5 shows that the data almost cover two cycles of the quasi-periodic frequency variations. While clearly not strictly sinusoidal, although not very far from it, if we fit the closest pure sine wave to each curve, we find that all have a very similar (quasi-)period of $\sim 570$ days. The variations for the side components (retrograde and prograde modes) are clearly in antiphase. For the amplitude variations, we also find suggestions of a quasi-periodic modulation for the central and prograde components. The retrograde mode for its part has a more regular amplitude evolution (increase) during the course of the observations.

Figure 6 illustrates the amplitude and frequency modulations for the $g$-mode triplet $T_{2}$ near $316 \mu \mathrm{Hz}$ using the same presentation as in Fig. 4. In this case, the triplet shows a very small (but significant) asymmetry of $0.0036 \mu \mathrm{Hz}$. The frequencies appear to be stable over the 38-month Kepler observations. The amplitude is essentially constant for the retrograde $(m=-1)$ mode while the other two components display some variations. The central one may show a small oscillatory behavior, but more precise measurements would be needed to really confirm that trend. The prograde $(m=1)$ mode has its amplitude rising continuously throughout the observations, from $400 \mathrm{ppm}$ up to about $600 \mathrm{ppm}$.

The amplitude and frequency variations of the $g$-mode triplet $T_{3}$ near $519 \mu \mathrm{Hz}$ are shown in Fig. 7. In this triplet, which

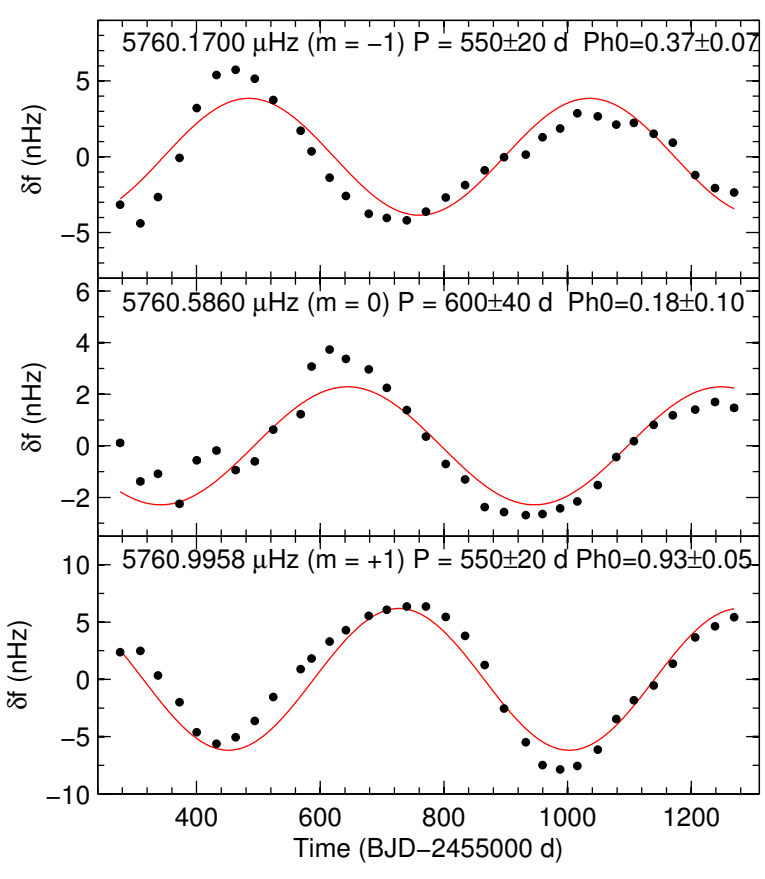

Fig. 5. Frequency modulations after removing the long-term trend in the $T_{1}$ triplet by applying a second-order polynomial fit. The solid curves represent the best fits of one pure sine wave to the frequency modulations. The associated formal errors for the periods and phases are also estimated. 

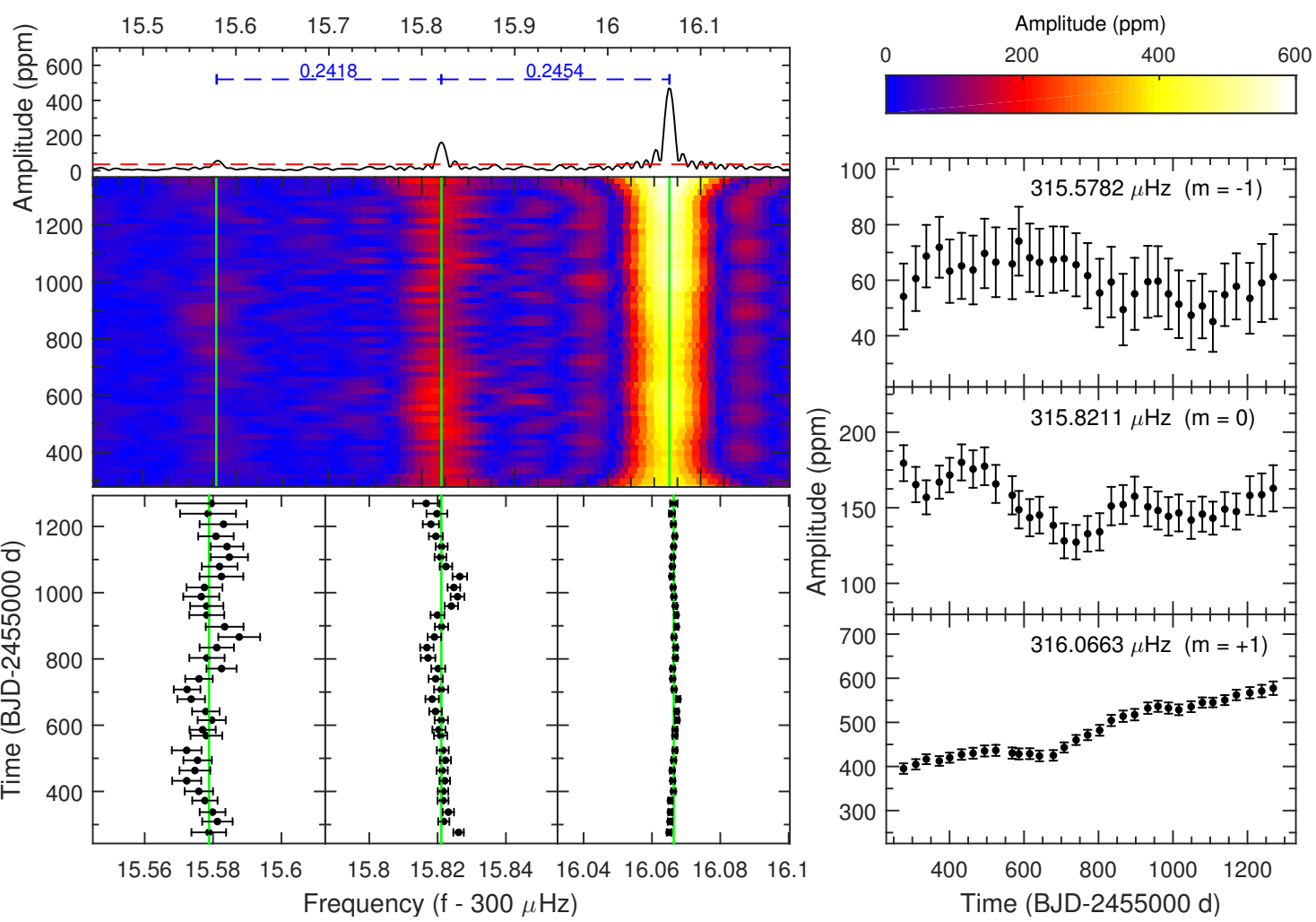

Fig. 6. Same as Fig. 4 but for the $T_{2} g$-mode triplet near $316 \mu \mathrm{Hz}$.

is almost perfectly symmetric, the three components have stable frequencies and amplitudes within the quoted uncertainties. This stability is further illustrated with Fig. 8 that shows the scattering of the measured frequencies and amplitudes for these modes from all the data chunks considered throughout the entire light curve. Almost all measurements are indeed confined within $2 \sigma$ around their average values (and all are within $3 \sigma$ ). It is interesting to note that the triplet $T_{3}$ therefore has different characteristics compared to the two triplets $T_{1}$ and $T_{2}$.

Figure 9 shows the amplitude and frequency modulations for the $g$-mode doublet $D_{1}$ near $394 \mu \mathrm{Hz}$. The frequencies of each component forming the doublet appear to be stable over the 38-month Kepler observations, while the amplitudes show very suggestive indications of quasi-periodic variations. We find that the amplitude modulations of the two components have very similar periods, about 1100 days, as illustrated by the best-fit sine waves to the data (the red solid curves in right panel of Fig. 9). Hence the available Kepler data just cover about one cycle of this variation, but it is remarkable that almost all the amplitude measurements match very closely the fitted sine curves. This estimated period is almost twice the period of modulations occurring in the main triplet $T_{1}$. Moreover, we clearly see that the amplitudes of the two components evolve almost in antiphase during the observing run.

Figure 10 shows the amplitude and frequency modulations for the $p$-mode quintuplet $Q_{1}$ near $5287 \mu \mathrm{Hz}$. In this complete quintuplet, the $m= \pm 2$ modes and possibly the $m=0$ central component show significant frequency variations. The other modes, with $m= \pm 1$, have frequencies which are rather stable (with only marginal fluctuations) over the entire observation run. In contrast, the amplitudes for all the modes in the quintuplet vary with patterns that cannot clearly be connected to periodic modulations, based on the available data. Of course, quasi periodic modulations with a timescale longer than twice the present
Kepler observation cannot be ruled out. We also note that the frequency variations of the $m=-2$ and $m=+2$ components and the amplitude modulations of the $m=-2$ and $m=-1$ components are roughly in antiphase during the observation.

Figure 11 shows the amplitude and frequency modulations for the $\ell>2 p$-mode multiplet $M_{1}$ near $5413 \mu \mathrm{Hz}$. The $\ell$-value for this group of modes is not clearly assessed yet, but a plausible interpretation is that it corresponds to an $\ell=4$ nonuplet (Baran et al. 2012) with three undetected components and one component barely visible in the LSP of the full data set (see topleft panel of Fig. 11) but which is too low in amplitude to be studied in subsets of the light curve. Some of the frequencies and amplitudes of the five clearly visible modes in this multiplet show significant variations during the 38 months of Kepler observation. In particular, the frequencies of the side components drifted toward each other by $\sim 0.032 \mu \mathrm{Hz}$ from the beginning to the end of the run. This trend may be comparable to that observed in the side components of the $T_{1}$ triplet (see Fig. 4). Moreover, the same phenomenon also occurs for some modes observed in the long-period-dominated sdB pulsator KIC 2697388 (M.D. Reed, priv. comm.).

In addition to the six multiplets discussed above, we identified other possible multiplets in the data, such as six modes near $5571 \mu \mathrm{Hz}$ (see Table A.1), in which however most of the components have amplitudes too low to be well studied in a timefrequency analysis or by prewhitening shorter parts of the light curve. We therefore do not consider them further in this work.

Beyond the multiplets generated by the rotation of the star, we also focus on the interesting narrow frequency region near $6076 \mu \mathrm{Hz}$ where three structures $\left(f_{23}, f_{35}\right.$ and $\left.f_{74}\right)$ show amplitude and frequency modulations as illustrated in Fig. 12. These frequencies are in fact related to components of the $T_{1}$ and $T_{2}$ triplets through linear combinations. We find that $f_{23} \sim f_{1}+f_{11}$, $f_{35} \sim f_{3}+f_{21}$, and $f_{74} \sim f_{3}+f_{11}$. Interestingly, we note that 

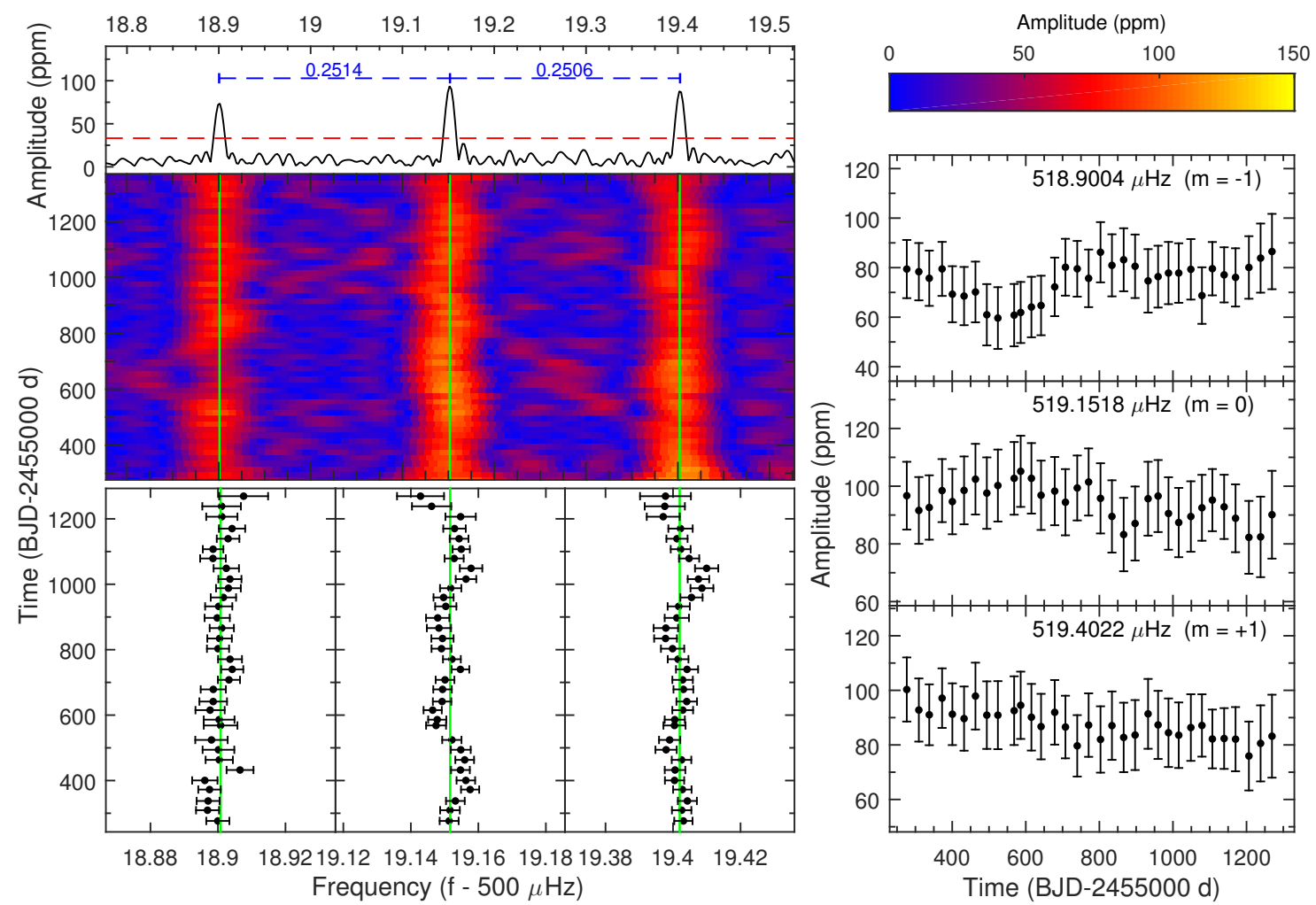

Fig. 7. Same as Fig. 4 but for the $T_{3} g$-mode triplet near $519 \mu \mathrm{Hz}$.
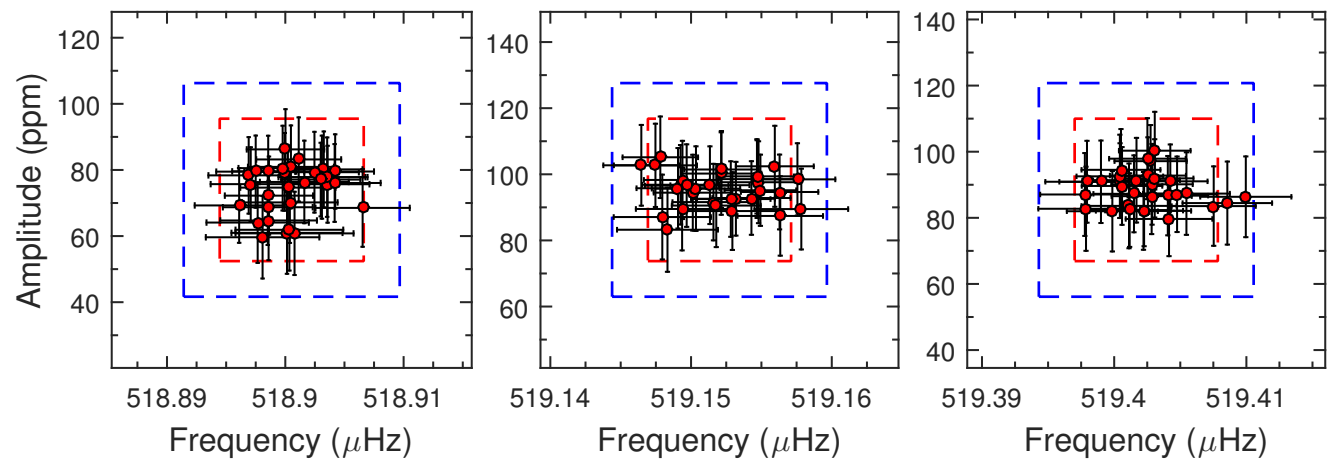

Fig. 8. Frequency and amplitude scattering for the three components forming the $g$-mode triplet $T_{3}$ around their averaged values. The red and blue dashed rectangles indicate the $2 \sigma$ and $3 \sigma$ error boxes, respectively. All the data points are within $3 \sigma$.

the frequency and amplitude variation pattern of $f_{23}$ is similar to the variations observed for the mode $f_{1}$. Similarities also exist between the variations observed in $f_{35}$ and $f_{2}$. The peak $f_{74}$, for its part, shows a rather large frequency variation covering up to $\sim 0.1 \mu \mathrm{Hz}$ (the scales of these frequency variations are indicated by the green shadowed region in the top panel of Fig. 12).

After this description of the various behaviors encountered, we concentrate, in the following sections, on plausible theoretical interpretations for the observed amplitude and frequency modulations.

\section{Resonant mode coupling and amplitude equations}

In this section, we recall the most natural theoretical background to understand the behavior of the modes forming the six multiplets induced by stellar rotation. These are indeed prone to develop nonlinear interactions through resonant mode couplings, which is the mechanism that we ultimately support from our present analysis. But before moving forward in discussing details on the nonlinear resonant coupling mechanism, we first rule out several other possibilities as the cause of the observed modulations, such as instrumental effects, binarity, stochastically driven pulsations, or stellar "weather".

Instrumental modulations can possibly occur, for example, on a per quarter basis, such as a slightly varying contamination from nearby stars that could affect the amplitude of the modes. Such effects would however affect all frequencies similarly, which is not what is observed with KIC 10139564 where the modes show different types of behavior. Another effect related to the instrument that could induce frequency and amplitude modulations is the slight shift of the Nyquist frequency associated with the movement of the Kepler spacecraft in the Solar System barycentric reference frame. Fortunately, the multiplets that we consider (with frequencies below $5761 \mu \mathrm{Hz}$ ), are far away from the Nyquist frequency limit $(\sim 8496 \mu \mathrm{Hz})$. Moreover, such 

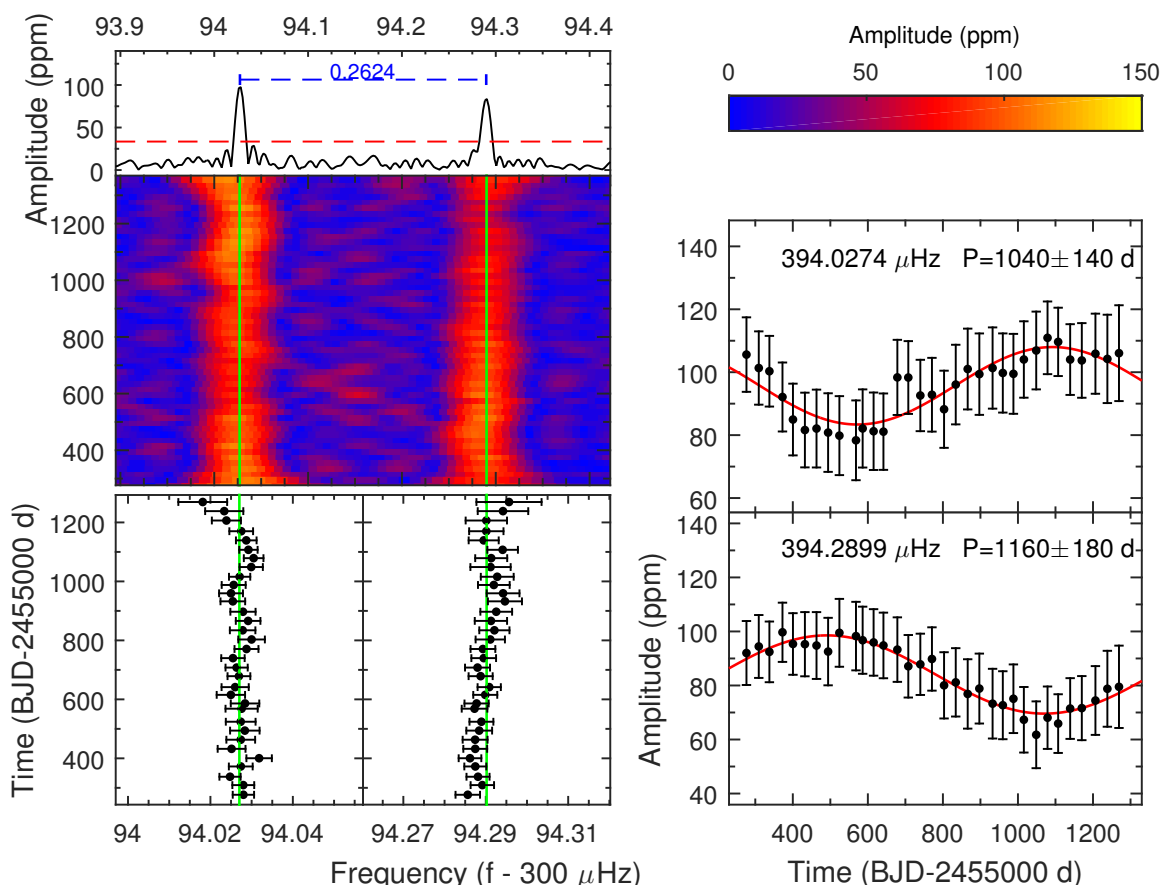

Fig. 9. Same as Fig. 4 but for the $D_{1} g$-mode doublet near $394 \mu \mathrm{Hz}$. The solid curves in right panel show the best fit of a pure sine wave to the amplitude modulations.
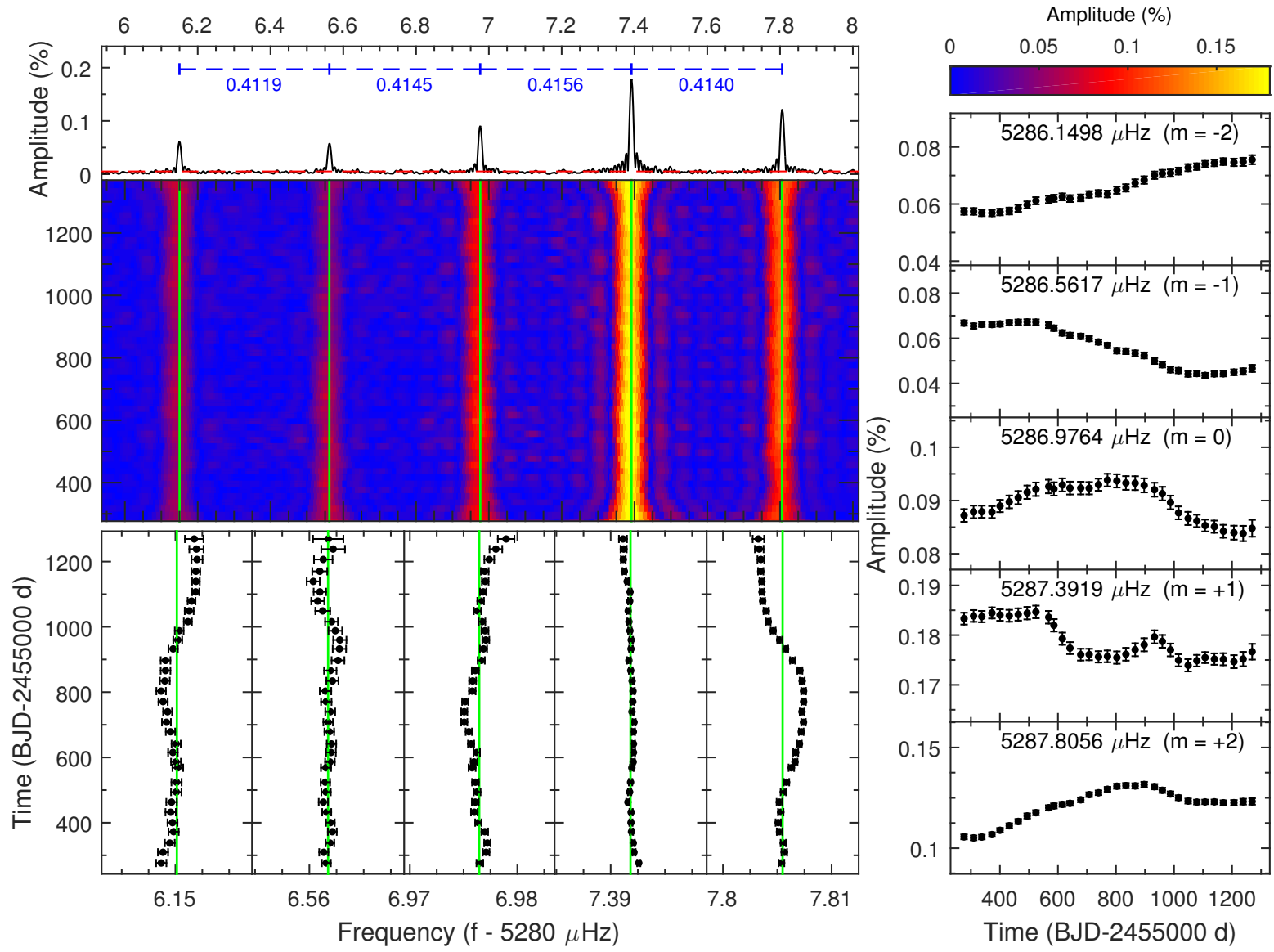

Fig. 10. Same as Fig. 4 but for the $Q_{1} p$-mode quintuplet near $5287 \mu \mathrm{Hz}$.

well-structured nearly equally spaced multiplets can obviously not be the mirror reflected frequencies of signals occurring above the Nyquist limit (Baran et al. 2012).

The presence of orbiting companions around compact stars could also induce frequency variations. However, these should occur in all frequencies and be correlated in phase, such as in the sdB pulsator V391 Peg (Silvotti et al. 2007). The variations that we find in several frequencies of KIC 10139564 are clearly not correlated in phase. In addition, radial velocity measurements from spectroscopy do not show any significant variation, thus 

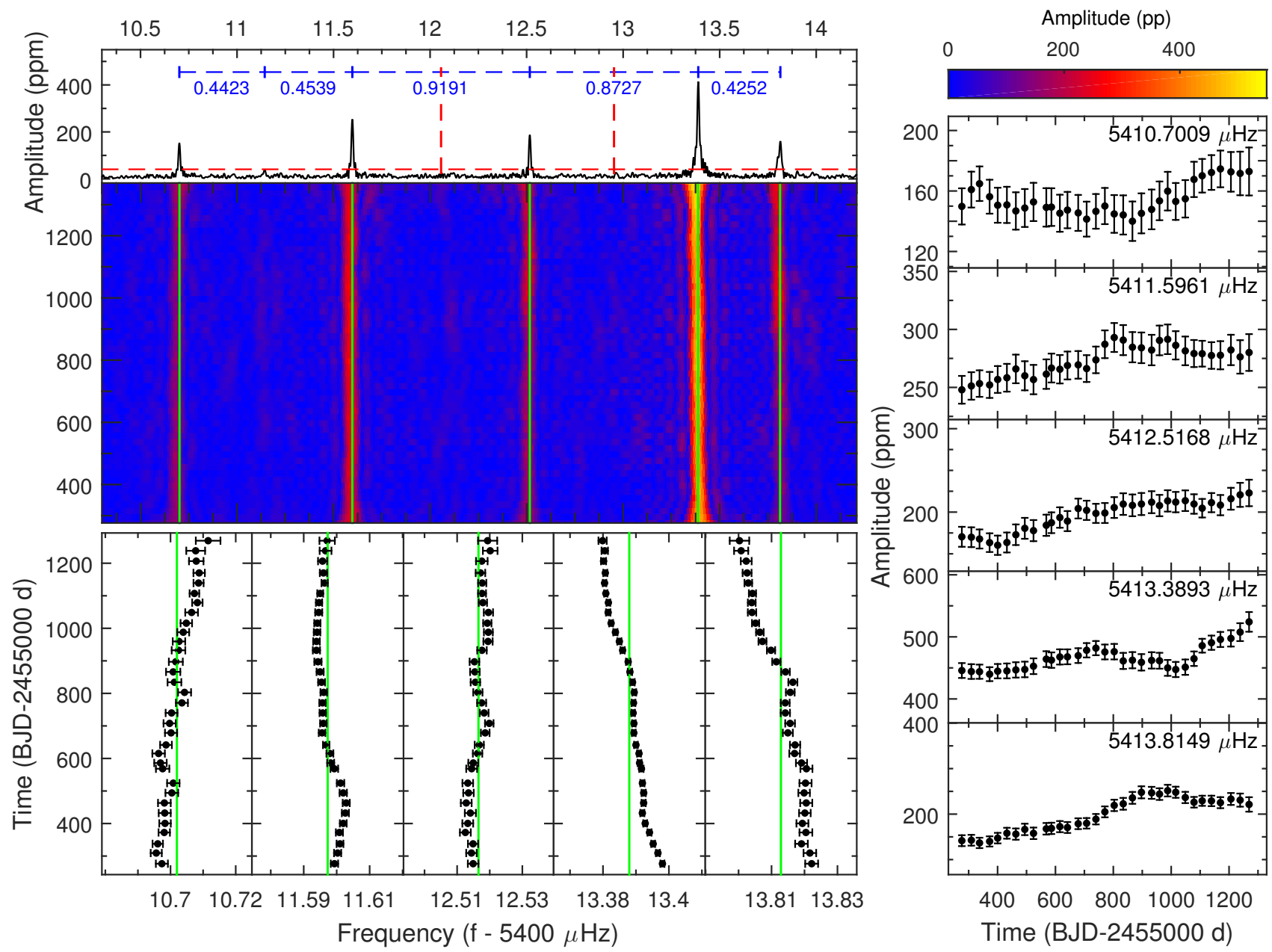

Fig. 11. Same as Fig. 4 but for the $\ell>2 p$-mode multiplet $M_{1}$ near $5413 \mu \mathrm{Hz}$. Note that at least three components are missing in this multiplet and the red vertical dashed lines indicate the expected position for two of them.

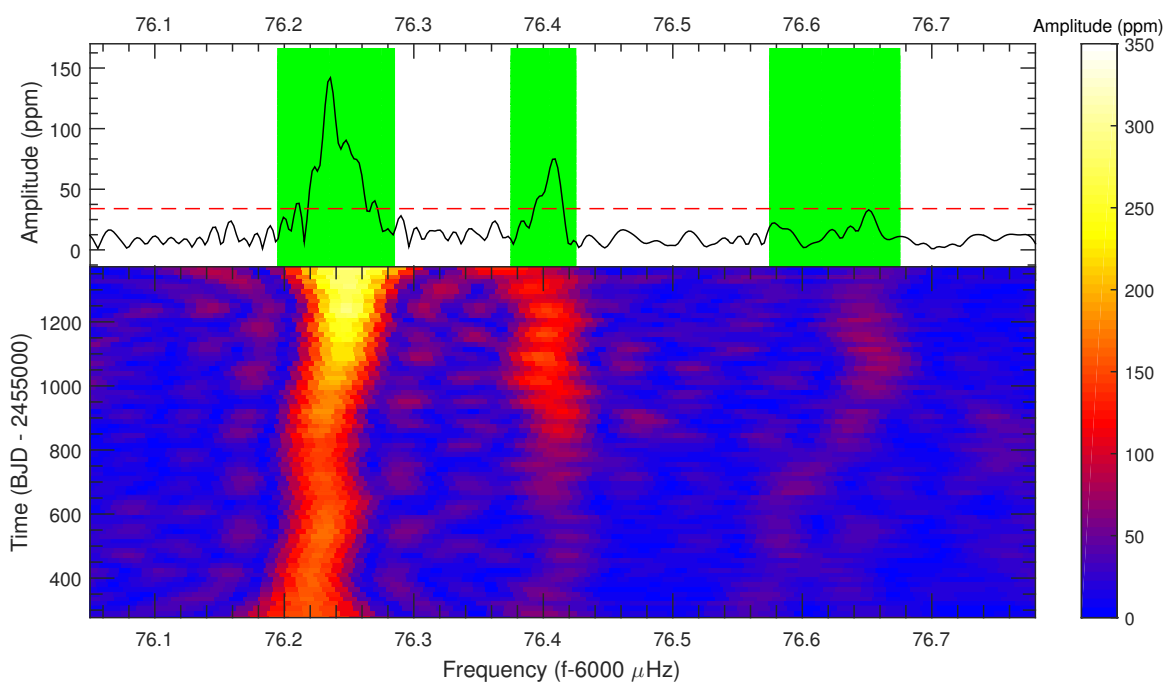

Fig. 12. Frequency and amplitude modulations of a group of linear combination frequencies $C_{1}$ near $6076 \mu \mathrm{Hz}$. The red dashed line indicates the $5.6 \sigma$ detection threshold. The green shadowed areas in top panel represent the scales of variation of these frequencies (see text for details). ruling out the presence of a stellar companion (Baran et al. 2012, but for substellar objects, a higher precision would be needed to exclude this possibility).

Stochastically driven pulsations by envelope convection have long been observed in the Sun and solar-like stars. It has been claimed in the past that stochastic oscillations could also occur in some sdB pulsators, based on the observation that mode amplitudes could vary from season to season (Kilkenny et al. 2010; Reed et al. 2007). Østensen et al. (2014) recently announced that stochastic pulsations were found in the $\mathrm{sdB}$ star KIC 2991276, in which the amplitude and phase of the modes vary substantially and irregularly on a timescale of a month. 
However, the mechanism responsible for the oscillations in sdB stars, a well identified $\kappa$-effect involving iron-group elements (Charpinet et al. 1997; Fontaine et al. 2003), is very different in nature from the stochastic driving occurring in the convective envelope of solar-like stars. Such a mechanism would indeed hardly be efficient in $\mathrm{sdB}$ stars that have radiative envelopes, except may be for a very narrow convective layer generated by the accumulation of iron in the Z-bump region (the latter being however extremely weak). Beyond these theoretical considerations, we find in the case of KIC 10139564 that several mode behaviors, for example, the frequencies in the triplet $T_{1}$ and the amplitudes in the doublet $D_{1}$, show correlations that would be difficult to account for with a stochastic driving mechanism and that essentially rule out this interpretation.

Changes in the background physical state of the star such as possibly induced by magnetic cycles could also be invoked for explaining amplitude and frequency modulations. Magnetic cycles indeed have an impact on the frequencies of $p$-modes observed in the Sun and lead to small frequency drifts that correlate in time with tracers of the solar surface activity (see, e.g., Salabert et al. 2015, and references therein). However, there is no clear observational evidence of stellar activity on the surface of $\mathrm{sdB}$ stars which, again, have very stable radiative envelopes and are not known to be magnetic. Cycles comparable to those observed in solar-like stars are therefore unlikely to be found in sdB stars. Moreover, such a mechanism, or more generally a phenomenon modifying the physical state of the star on a timescale of months could hardly account for the observed modulations in KIC 10139564 that show very different modulation behaviors from mode to mode, while a global change in the star would affect all modes similarly. Consequently, we also rule out this possibility in the present case.

\subsection{Triplet resonance induced by slow stellar rotation}

We hereafter propose that nonlinear resonant coupling mechanisms could be a natural explanation for the observed modulations in KIC 10139564. Resonant interactions between modes may indeed result in amplitude and frequency variations occurring on timescales of weeks, months, and even years.

In the present context, we limit ourselves to the type of resonances described in Buchler et al. $(1995,1997)$ involving linear frequency combinations $v_{1}+v_{2} \sim 2 v_{0}$. More specifically, we focus on a particular case where dipole $(\ell=1)$ modes are split by slow rotation and form a nearly symmetric triplet (thus following the above relationship between the frequencies of the components). We also consider the three-mode couplings of the form $v_{1}+v_{2} \sim v_{0}$, which corresponds to the so-called direct resonances or parametric instabilities (Dziembowski 1982; Wu \& Goldreich 2001).

We first recall some basic theoretical background relative to resonances in mode triplets created by stellar rotation. We emphasize that our focus on this particular mechanism is obviously motivated by the specific configuration of the modes observed in KIC 10139564, most of which are identified as $\ell=1$ rotationally split triplets. We also point out that this type of nonlinear resonance has recently been strongly suggested to explain the modulations of the $g$-modes triplets in the DB white dwarf KIC 08626021 (Z16). It is therefore the most natural effect that one could think of in the present case. The AEs formalism could also, in principle, be extended to multiplets of degree $\ell>1$ at the expense of solving a larger set of coupled amplitude equations
(Buchler et al. 1995). However, such development has yet to be done, which is beyond the scope of our present paper. The latter would be needed for KIC 10139564 in order to fully interpret the several multiplets with $\ell>1$ that show variations. The behavior for more complex $\ell>1$ multiplets may indeed differ from the simpler (better documented) $\ell=1$ triplet case, although we expect some similarities in general.

Details on the theory of nonlinear resonant couplings for three-mode interactions, such as in $\ell=1$ triplet, can be found in (Buchler et al. 1995, 1997, Z16). We summarize below the most relevant aspects (for our purposes) of the theory. In particular, The quantity $\delta v$ (which we thereafter call the frequency asymmetry), measuring the departure from exact resonance (that would occur if, e.g., triplets were perfectly symmetric), is in fact essential for driving the various resonant mode coupling behaviors. Contributions to the frequency asymmetry in a given triplet generally involves higher order effects of stellar rotation on the pulsation frequencies (Dziembowski \& Goode 1992; Jones et al. 1989), but could also have additional origins, such as the presence of a weak magnetic field ${ }^{2}$. We do not however consider further that possibility since no evidence of significant magnetism exists for sdB stars (Petit et al. 2012; Landstreet et al. 2012).

The rotationally split frequencies up to the second order, which should be the main contribution to the frequency asymmetry, are given by the formula

$v_{m}-v_{0}=\left(1-C_{k \ell}\right) m \Omega+D_{k \ell} \frac{m^{2} \Omega^{2}}{v_{0}}$,

where the $C_{k \ell}$ coefficient is the well-known first order Ledoux constant, $D_{k \ell}$ involves a complex integration of the eigenfunctions of the modes, and $\Omega=1 / P_{\text {rot }}$ is the rotation frequency of the star (expressed in Hertz). The value of $C_{k \ell}$ is typically $\sim 0.5$ for dipole $g$-modes when approaching the asymptotic regime, while it is usually very small $\left(C_{k \ell} \ll 1\right)$ for $p$-modes. The second order coefficient $D_{k \ell}$ is roughly $4 C_{k \ell}$ for dipole $g$-modes (Dziembowski \& Goode 1992; Goupil et al. $1998)$ but can vary significantly from one $p$-mode to another (Dziembowski \& Goode 1992; Saio 1981). The rotation period of KIC 10139564, $P_{\text {rot }}$, can be estimated from the average of the frequency separations between the components of the multiplets using the first order approximation $\Delta v=\left(1-C_{k \ell}\right) \Omega$. We find $P_{\text {rot }} \sim 26$ days for KIC 10139564 (see Sect. 2). An "observed" frequency asymmetry can also be evaluated directly from the measured frequency of each triplet component, simply from the relation

$\delta v_{\mathrm{o}}=v_{-}+v_{+}-2 v_{0}$.

We note at this stage that $\delta v_{\mathrm{o}}$ may actually differ from asymmetries expected from linear developments (such as discussed above) because nonlinear effects can modify the frequencies of the modes.

The numerical solutions of the AEs for mode interactions in triplets mainly reveal three distinct regimes of resonances (see, e.g., Buchler et al. 1997, and in Z16). The first state is the "frequency lock" regime where all the components in the triplet have constant frequencies and amplitudes and the asymmetry tend to be zero (triplets become perfectly symmetric). The opposite configuration is the nonresonant regime where the triplet

2 The asymmetry would be proportional to the strength of magnetic field $|\boldsymbol{B}|^{2}$ and the frequency of each component of the triplet (except the central, $m=0$, one) would be shifted in the same direction (Jones et al. 1989). 
configuration is likely predicted by the linear theory of stellar oscillations. Between the two, there is an intermediate regime in which all the modes in the triplet show modulated frequencies and amplitudes which can be periodic, irregular, or even chaotic.

In order of magnitude, the occurrence of these three regimes is roughly linked to a parameter $D$ defined as (see Goupil et al. 1998)

$D \equiv \frac{2 \pi \delta v}{\kappa_{0}}$

where $\kappa_{0}$ is the linear growth rate of the $m=0$ mode in the triplet (a nonadiabatic quantity). However, the ranges of values for this parameter which define the different regimes depend somewhat on the values of the nonlinear coefficients in the real star. $D$ is also a quantitative indicator that measures how far the triplet modes are away from the exact resonance center $(D=0)$. We nonetheless summarize some of the properties encountered in previous studies as a function of $D$ :

- In the frequency-locked regime $(\delta v \rightarrow 0)$, the $D$-parameter roughly corresponds to values in the range $\sim 0-1$ according to the AEs formalism. However, in the case of the white dwarf star GD 358, Goupil et al. (1998) found that $D$ could be up to 20 and still correspond to a frequency-locked situation. These ranges, therefore, are somewhat dependent on the specific properties of the mode being considered, in particular on the scale of their linear growth rate, $\kappa_{0}$.

- The intermediate regime occurs when the triplet components move away from the resonance center $(\delta v \neq 0)$. In this situation, if periodic variations indeed affect the considered modes, these can be expected to have a modulation timescale of

$P_{\text {mod }} \sim \frac{1}{\delta v} \simeq \frac{2 \pi}{\kappa_{0}} \frac{1}{D}$,

that is, roughly the timescale derived from the inverse of the linear (i.e., unperturbed) frequency asymmetry of the triplet dominated by the second order effect of stellar rotation (following Eq. (2)). This timescale is also connected to the inverse of the growth rate of the oscillation mode through the $D$ parameter (Goupil et al. 1998).

- The modes recover a configuration of steady pulsations with the nonresonant regime when the involved frequencies are such that the modes are now far from the resonance condition $(D \gg 1)$. In this regime, the nonlinear interaction between modes is very weak and nonlinear frequency shifts become very small. Consequently, the mode frequencies are close to the linear ones.

We finally point out that in addition to the above mentioned three main regimes, a narrow hysteresis (transitory) regime exists between the frequency-locked and intermediate regimes in which the frequencies can be locked (i.e., constant), while the amplitudes still have a modulated behavior (Buchler et al. 1997).

\subsection{Three mode resonance of the type $v_{0} \sim v_{1}+v_{2}$}

In this section, we recall some properties of nonlinear interactions between oscillations modes not within triplets but whose frequencies are close to a resonance condition such that $v_{0} \sim$ $v_{1}+v_{2}$. Frequencies with such a relationship could also result from simple linear combination frequencies, that is, exact sum or difference of frequencies (where the "child" frequency is not a true eigenmode), which may be related to nonlinearities in the mixing process affecting the depth of a convective zone in the outer layer of a pulsating star (Wu 2001), or to nonlinearities in the flux response induced by the surface geometrical and temperature distortions triggered by the propagating waves (Brassard et al. 1995).

A useful quantity, $R$, connecting the observed amplitude of the combination frequency and the amplitudes of its "parent" modes, has been defined as (van Kerkwijk et al. 2000; Wu 2001)

$R=\frac{A_{0}}{A_{1} \cdot A_{2}}$,

where the $A_{0}, A_{1}$, and $A_{2}$ are the amplitudes of the frequencies $v_{0}, v_{1}$, and $v_{2}$, respectively. This ratio $R$ is typically less than ten for simple linear combinations related, such as, to nonlinearities in the flux response. Consequently, in pulsating sdB stars, the "child" frequency resulting from this effect usually has a very low amplitude compared to its "parent" frequencies. In the large amplitude and brightest known pulsating sdB star, Balloon 090100001, where such linear combination frequencies have been unambiguously observed, the amplitude ratios are 3.9, 3.7, 3.0 and 5.5 for the linear combination frequencies of four $p$-modes and one $g$-mode $f_{1}+f_{2}, f_{1}+f_{3}, f_{1}+f_{4}$ and $f_{1}-f_{B}$ in $B$-band photometry (Baran et al. 2008), respectively. In the present work, we however find that the identified linear combination frequencies $C_{1}$ have amplitude ratios in the 10-100 range, that is, one order of magnitude larger than typical linear combination frequencies observed so far. One possible interpretation for the high amplitude ratios is that the frequency sum/difference is near the resonance condition of $v_{0} \sim v_{1}+v_{2}$ and its amplitude is boosted significantly by the resonance (e.g., Dziembowski 1982; Breger \& Montgomery 2014).

The AEs formalism treating the $v_{0} \sim v_{1}+v_{2}$ type of resonance, including the parametric instability and the direct resonance (see below), is similar to the case of a triplet resonance (e.g., see the amplitude equations in Buchler et al. 1997, Z16). According to Dziembowski (1982), the three-mode interactions can be described by the following coupled system

$$
\begin{aligned}
\frac{\mathrm{d} \boldsymbol{A}_{\mathbf{0}}}{\mathrm{d} t} & =\kappa_{0} \boldsymbol{A}_{\mathbf{0}}+\mathrm{i} \frac{q}{2 v_{0} I_{0}} \boldsymbol{A}_{\mathbf{1}} \boldsymbol{A}_{\mathbf{2}} \exp (-\mathrm{i} \delta v t), \\
\frac{\mathrm{d} A_{1}}{\mathrm{~d} t} & =\kappa_{1} \boldsymbol{A}_{\mathbf{1}}+\mathrm{i} \frac{q}{2 v_{1} I_{1}} \boldsymbol{A}_{\mathbf{0}} \boldsymbol{A}_{\mathbf{2}}^{*} \exp (-\mathrm{i} \delta v t), \\
\frac{\mathrm{d} \boldsymbol{A}_{\mathbf{2}}}{\mathrm{d} t} & =\kappa_{2} \boldsymbol{A}_{\mathbf{2}}+\mathrm{i} \frac{q}{2 v_{2} I_{2}} \boldsymbol{A}_{\mathbf{0}} \boldsymbol{A}_{\mathbf{1}}^{*} \exp (-\mathrm{i} \delta v t) .
\end{aligned}
$$

Where $\boldsymbol{A}_{j}^{*}$ is the complex conjugate of the amplitude $\boldsymbol{A}_{j}\left(\boldsymbol{A}_{j}=\right.$ $\left.A_{j} \mathrm{e}^{\mathrm{i} \phi_{j}}\right), I_{j}$ is the mode inertia, and $\kappa_{j}$ is the linear growth rate for the three involved frequencies. The quantity $q$ is a nonlinear coupling coefficient, and $\delta v$ is the frequency mismatch relative to pure resonance defined by the relationship $\delta v=v_{0}-v_{1}-v_{2}$.

The nonlinear Eqs. (6a), (6b) and (6c) cannot be solved by analytical methods, but solutions for the equilibrium state (all time derivatives set to zero) can be obtained. In particular, the equilibrium solution leads to an amplitude ratio

$$
R=\frac{q}{2 v_{0} K_{0} I_{0}} \equiv \frac{A_{0}}{A_{1} \cdot A_{2}}
$$

The stability of the equilibrium-state depends on the growth (damping) rates and the frequency mismatch (e.g., Dziembowski 1982). 
In a three-mode direct resonance, the child mode is damped and has a frequency very close to the sum of frequencies of its two parent modes which are linearly driven (unstable). The child mode amplitude is very sensitive to its mode inertia, linear growth rate, and to the nonlinear coupling coefficient (see Eq. (7)). This near resonance mode can grow up to a very large amplitude if the quantity $q / \kappa_{0} I_{0}$ is sufficiently large. The coupling coefficient $q$ follows from a complex integration of the coupled mode eigenvectors and its explicit form can be found in Dziembowski (1982). It may be possible, in principle, to calculate this coefficient provided that the mode eigenfunctions are known. However, this would require that a precise seismic solution is found for KIC 10139564, which still has to be obtained. To test the theory of a three-mode direct resonance, we would also need to know the linear damping (growth) rate and the inertia of the damped mode, eventually corrected by the effect of slow stellar rotation (e.g, see Carroll \& Hansen 1982). However, the situation could be simplified in the case of unstable equilibrium state where the amplitude and frequency of the child mode should exactly follow those of its parent modes, even if the growth rates and coupling coefficients are unknown. Fortunately, the equilibrium state of three-mode direct resonances seems always unstable because the growth (damping) rates cannot satisfy the stability criteria of the Hurwitz theorem (Dziembowski 1982). Therefore, each frequency and amplitude measurement could be used as one independent test of these particular nonlinear couplings. Furthermore, this also provides a method to separate the child mode from their parent modes according to the amplitude and frequency relationships (e.g., Breger \& Montgomery 2014).

The parametric instability is another form of three-mode resonant coupling that could destabilize a pair of stable daughter modes from an overstable (driven) parent mode (Wu \& Goldreich 2001). In this mechanism, the overstable parent mode gains energy through the driving engine (a $\kappa$-mechanism in our case) and the two independent damped child modes dissipate energy. This configuration would lead the system to reach limit cycles under certain conditions (e.g., if $\delta v<\kappa_{1}$ or $\kappa_{2}$, Wu \& Goldreich 2001; Moskalik 1985). During such limit cycles, the amplitude of the parent mode first increases slowly on a timescale of $\kappa_{0}^{-1}$, then decreases rapidly on a timescale of $\kappa_{1,2}^{-1}$. A the same time, the amplitude of the daughter modes follow the opposite behavior. In sdB stars, we point out that the linear growth rate of the parent mode $\kappa_{0}$ would be usually far smaller than the damping rate of the daughter modes $\kappa_{1,2}$. We further mention that the nonlinear interactions between the parent/child modes would also affect their periods as a result of phase variations. The nonlinear frequency shift could be of the order of a few $\mu \mathrm{Hz}$ in some extreme conditions (Wu \& Goldreich 2001; Moskalik 1985). We point out that a parametric instability can also occur in multiplets. In such circumstances, different $m$ components that forms the multiplet may share some common damped daughter modes. Having common daughter modes involved in different parametric resonances, that is, involving different parent overstable components, will obviously induce more complex dynamic modulations than simple periodic variations that could be expected from pure three-mode only interactions. We indeed point out that both the triplet resonance that was explored by Buchler et al. $(1995,1997)$ and the three-mode $v_{0} \sim v_{1}+v_{2}$ resonances that were investigated by Dziembowski (1982), Moskalik (1985) and Wu \& Goldreich (2001) are treated as isolated systems, i.e., assuming only interactions between the three involved modes and ignoring the possible influence of other modes. Modes with the highest amplitudes are more likely to efficiently couple with different resonances, such as in a multiplet resonance and in a $v_{0} \sim v_{1}+v_{2}$ resonance.

\section{Connections with mode behaviors seen in KIC 10139564}

In light of the theoretical background summarized in the last section, we tentatively interpret some of the behaviors described in Sect. 2 for the frequencies listed in Table 1. These indeed show striking similarities with patterns expected for nonlinear resonant mode interactions that occur in various regimes.

\subsection{Multiplets in the intermediate regime}

The first connection is for the modes belonging to multiplets that show quasi-periodic amplitude and frequency modulations. In particular, the $p$-mode triplet near $5760 \mu \mathrm{Hz}\left(T_{1}\right)$ shows indication that it could be evolving within the so-called intermediate regime of a triplet resonance. We recall (see Sect. 2; Fig.5) that the frequencies of the two side components in this triplet, besides showing a long term drift, vary quasi-periodically in antiphase with a timescale of $\sim 550$ days. The central component of $T_{1}$, for its part, has a frequency modulation which also vary, possibly with a slightly longer period of $\sim 600$ days. For comparison purposes, the modulation timescale is expected to be related to the inverse of the linear (i.e., unperturbed) frequency asymmetry in the triplet (see Eq. (4)), which therefore should be $\delta v \sim 0.02 \mu \mathrm{Hz}$. Assuming that this frequency asymmetry originally comes from the second order effect of slow rotation, and given the average rotation frequency of the star $(\sim 0.42 \mu \mathrm{Hz}$, corresponding to $\sim 26$ days), the $D_{k \ell}$ coefficient in Eq. (1) can be estimated to $\sim 200$ for that mode. This value is plausible because the $D_{k \ell}$ coefficient is found to vary over a large range for dipole $p$-modes (Dziembowski \& Goode 1992; Saio 1981). However, to compute $D_{k l}$ in this specific case and compare with this value, a precise seismic solution for KIC 1013956 has to be worked out, but is not available yet. It has to be noted that the frequency asymmetry measured from the averaged frequencies given in Table 1 is only $0.0026 \mu \mathrm{Hz}$ that is one order of magnitude lower than the value derived from the modulation frequency $(\sim 0.02 \mu \mathrm{Hz})$. We note, however, since the frequencies are varying with time, that the maximum extent of the observed frequency asymmetry is $\sim 0.02 \mu \mathrm{Hz}$ when considering the 33 measurements independently (see Fig. 4). We point out that these observed values $(0.0026 \mu \mathrm{Hz}$ on average and $\sim 0.02 \mu \mathrm{Hz}$ for the maximum asymmetry) are similar to those observed in the main triplet of the DBV star KIC 08626021, which is also in the intermediate regime (Z16). Nonlinear resonant interactions are bound to perturb the linear frequencies of the modes, forcing them in some cases to shift toward the exact resonance (obtained when the system is locked). It is therefore not surprising to observe a frequency asymmetry that can be significantly smaller than the theoretical shift expected in the linear theory context.

In terms of amplitude modulations, the situation is bit less clear as only the prograde component in $T_{1}$ shows a quasiperiodic modulation, with a timescale of $\sim 800$ days, while, for the other two components, particularly for the retrograde mode, their amplitude variations appear somewhat irregular.

In addition to the frequency variations of $T_{1}$ discussed above, we note that the three components that form this triplet feature a regular drift toward each other which, if nothing change, would lead them to merge into one frequency on a timescale of $\sim 10 \mathrm{yr}$. 
Such a merging is of course not conceivable and what we observe is more likely a small fraction of a variation cycle occurring on a timescale much longer than the duration of the Kepler observations. This suggests that the triplet resonance is probably not the only mechanism that affects the stability of $T_{1}$. This added complexity may also explain the more erratic behavior of the amplitude variations in this triplet. Quite notably, we indeed find that all the components of $T_{1}$ can be linked to other frequencies forming linear combinations satisfying the conditions for a three-mode resonance $v_{0} \sim v_{1}+v_{2}$. This will be further discussed in Sect. 4.4.

The quintuplet $Q_{1}$ also shows components with amplitude and frequency variations (see Fig. 10) that may be associated to the intermediate regime. In this case, however, we cannot estimate timescales for the modulations which appear to have a more complex behavior than the modulations detected in the $T_{1}$ triplet or, if we compare to other cases, in the triplets of the pulsating DB star KIC 08626021 (Z16). The averaged frequency mismatch, $\delta v_{\mathrm{o}}$, for $Q_{1}$ is about $0.0018 \mu \mathrm{Hz}$. This could either be the result of the nonlinear coupling mechanism locking the modulated components close to the exact resonance, even if they are in the intermediate regime (see the case of $T_{1}$, as well as the triplets in the DBV star KIC 08626021), or it could indicate that the modulation timescale for $Q_{1}$ is $\sim 17.6 \mathrm{yr}$ (the inverse to $\delta v_{\mathrm{o}}$, if their amplitudes have a periodic behavior). As there has been no theoretical exploration of the nonlinear five-mode interaction yet, the connection of $Q_{1}$ with the intermediate regime is based on the assumption that nonlinear five-mode interactions has also mainly three distinct regimes. The coupled amplitude equations for the five-mode resonance involve more terms in each $\mathrm{AE}$ and the numerical solutions are more difficult to search for.

Another case may be connected with the intermediate regime: the multiplet $M_{1}$ which shows amplitude and frequency variations (see Fig. 11). But, again, we cannot determine any timescale for the complex modulations occurring in this multiplet. In that case, there is also a slow trend leading frequencies, particularly for the most side components, to seemingly converge. This trend is very similar to the slow variation observed in the $T_{1}$ triplet. It could possibly be a fraction of a variation cycle with a much longer timescale than the duration of the observations, but more observations would be needed to confirm this hypothesis. This multiplet $M_{1}$ should be the siege of even more complex resonant coupling interactions than the quintuplet $Q_{1}$, since there are six detected components, with at least three components missing.

\subsection{Triplets in the transitory regime}

Another type of behavior encountered in our data can be linked to the narrow transitory hysteresis regime which is between the frequency lock and intermediate regimes. This state is characterized by stable frequencies but varying amplitudes. This is notably observed in the $g$-mode triplet $T_{2}$ (see Fig. 6). For this triplet, the observed frequency mismatch is about $0.0036 \mu \mathrm{Hz}$, that is, very similar to the value measured for the $T_{1}$ triplet (see left-top panel of Figs. 4 and 6). We also point out that $T_{2}$ may couple with the $p$-mode triplet $T_{1}$ through a three-mode resonance $v_{0} \sim v_{1}+v_{2}$, as discussed in Sect. 4.4.

The incomplete triplet $D_{1}$ may also be associated to this transitory regime as it shows quasi-periodic amplitude modulations and stable frequencies. Due to the missing component, we cannot measure the frequency mismatch for this doublet. We note that the AEs for the triplet resonance indicate that the modes cannot be stable, i.e, there is no fixed-point solution, if one of the visible modes forming the incomplete triplet is the central ( $m=0$ ) component (Buchler et al. 1995). Thus, at this stage, we may just fail to detect either the third component of the triplet whose amplitude may be lower than the detection threshold (meaning that the triplet is indeed in the narrow transitory regime), or the nonlinear modulation of the frequencies, which may be smaller in amplitude than our current precision (meaning a doublet in the intermediate regime, as predicted by the AEs).

\subsection{A triplet in the frequency-locked regime}

The last case occurring in a different regime is the $g$-mode triplet $T_{3}$, which shows stable amplitudes and frequencies (see Figs. 7 and 8 ). This suggests that $T_{3}$ is in the configuration of the frequency-locked regime where the triplet approaches the resonance center, i.e., $\delta v \rightarrow 0$ and both the frequencies and amplitudes are constant. Indeed, we find that the observed frequency asymmetry, $\delta v_{\mathrm{o}}$, is $0.0008 \mu \mathrm{Hz}$ (Fig. 7) for $T_{3}$, which is less than the measured error $0.0011 \mu \mathrm{Hz}$. The triplet $T_{3}$ is therefore exactly (within measurement errors) at the resonance center, contrary to $T_{1}$ and also $T_{2}$ which has constant frequencies but a small non-zero frequency mismatch.

In summary, the various behaviors encountered in the multiplets detected in KIC 10139564 seem to cover all the different regimes expected in a context of resonant mode coupling in multiplets. This mechanism is therefore quite likely responsible, at least in part, for the observed phenomena. In the following section, we discuss another type of resonance, the $v_{0} \sim v_{1}+v_{2}$ nonlinear interaction.

\subsection{Three-mode resonance}

In this section, we discuss the variations of a group of frequencies $C_{1}$, including $f_{23}, f_{35}$ and $f_{74}$, that are involved in a relationship $v_{0} \sim v_{1}+v_{2}$. We find that the variations of these frequencies have strong correlations with the variations of the components in the triplet $T_{1}$ (see Figs. 4 and 12). The large frequency variations first suggest that the $C_{1}$ frequencies correspond to three-mode resonances rather than simple linear combination frequencies. The result of prewhitening the frequencies $f_{23}$ and $f_{35}$ (using the same method as for the multiplets) is shown in Figs. 13 and 14, respectively. Most of the amplitude and frequency measurements for $f_{23}$ and $f_{35}$ are exactly following the variation of amplitude and frequency of the sums $f_{1}+f_{11}$ and $f_{3}+f_{21}$ within $1 \sigma$, respectively (see in particular the middle and bottom panels of Figs. 13 and 14).

The amplitude ratio $R$ is 37 and 85 for $f_{23} \sim f_{1}+f_{11}$ and $f_{35} \sim f_{3}+f_{21}$, respectively. These values are significantly higher than those observed for normal linear combination frequencies in sdB stars (see the example given in Sect. 3.2). There is also a possible true linear combination frequency in KIC 10139564 with the frequency $f_{79} \sim f_{1}-f_{4}$ (see Table 2 ) which indeed has a very low amplitude (signal-to-noise ratio of 5.1) and an amplitude ratio $R$ less than one. Thus, we propose that there should be real pulsation modes near the position of the linear combination frequencies $f_{1}+f_{11}$ and $f_{3}+f_{21}$ and these modes had their amplitudes boosted through a resonance. In the $v_{0} \sim v_{1}+v_{2}$ resonance, the child mode indeed follows the behavior of its parent modes (see, again, Figs. 13 and 14 and examples provided by Breger \& Montgomery 2014).

We note that another frequency, $f_{74}$, is also in the region near $6076 \mu \mathrm{Hz}$, but has an amplitude too low to be monitored over time using the prewhitening technique on subsets of the 

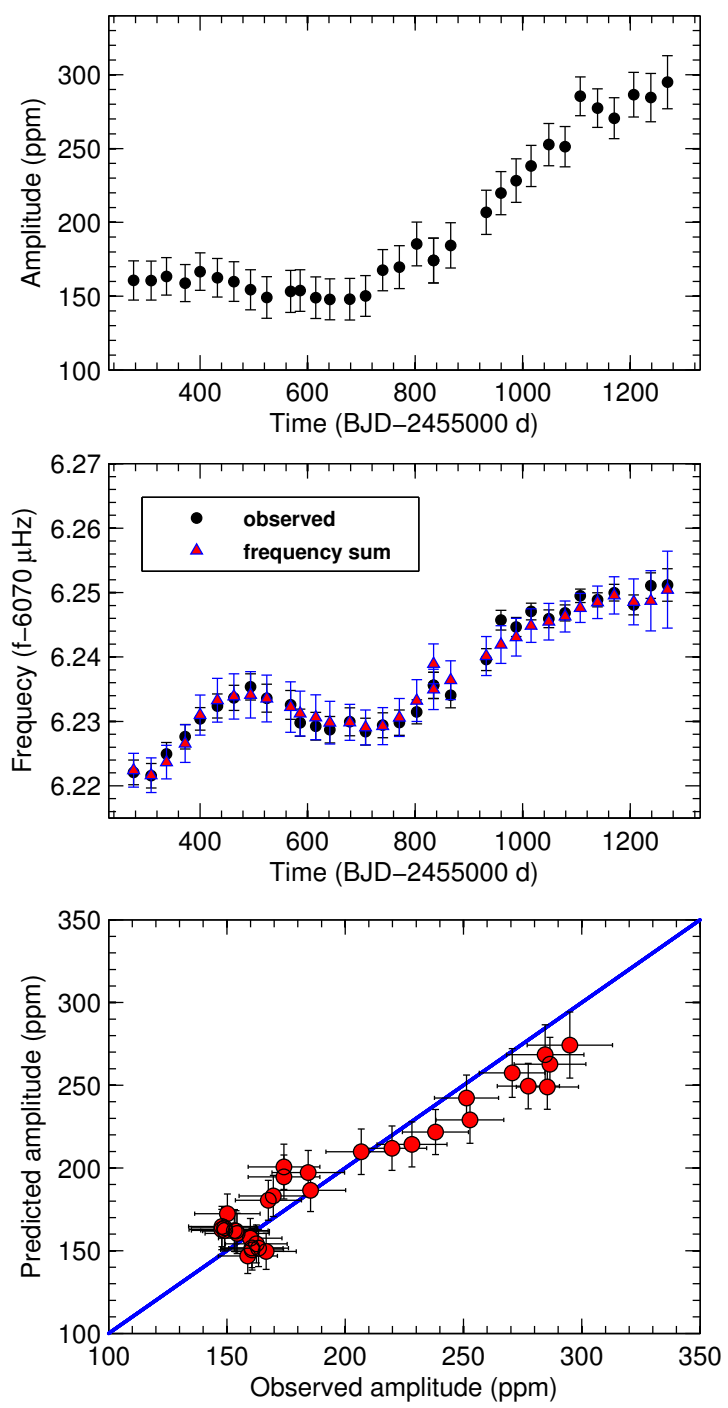

Fig. 13. Amplitude and frequency variations of the linear combination frequency $f_{23}=f_{1}+f_{11}$. Top panel: measured amplitudes as a function of time obtained from each data subset (using the same method as for multiplets). Middle panel: measured frequencies from each data subset compared with the frequency sum $f_{1}+f_{11}$, both as a function of time. Bottom panel: observed amplitudes of $f_{23}$ vs predicted amplitudes of $R \times$ the product of $f_{1}$ and $f_{11}$ amplitudes (see Eq. (6) for the definition of $R)$. In both cases (frequency and amplitude comparisons), the measurements are found to be within $1 \sigma$.

data. However, Fig. 12 still clearly shows that this frequency is varying smoothly during the observation, from $\sim 6076.58 \mu \mathrm{Hz}$ (the first half part of the run) to $\sim 6076.68 \mu \mathrm{Hz}$ (the second half part). We speculate that there is possibly a real mode, with a frequency around $6076.58-6076.69 \mu \mathrm{Hz}$, which first interacts with the frequency sum $f_{2}+f_{39} \sim 6076.59 \mu \mathrm{Hz}$, then with the frequency sum $f_{3}+f_{11} \sim 6076.66 \mu \mathrm{Hz}$, because the influence from the latter modes become stronger than the former ones during the last half of the observation run, due to the amplitude of $f_{3}$ increasing significantly in the second half of the Kepler time series. This, again, suggests that the $C_{1}$ frequencies are really part of $v_{0} \sim v_{1}+v_{2}$ resonances instead of being simple linear combination frequencies.

All of the involved frequencies, $f_{1,2,3,11,21,39}$, are the components of the triplets $T_{1}$ and $T_{2}$. They are expected to be overstable (driven) modes, thus meaning that they are involved in a three-mode direct resonance and not a parametric resonance
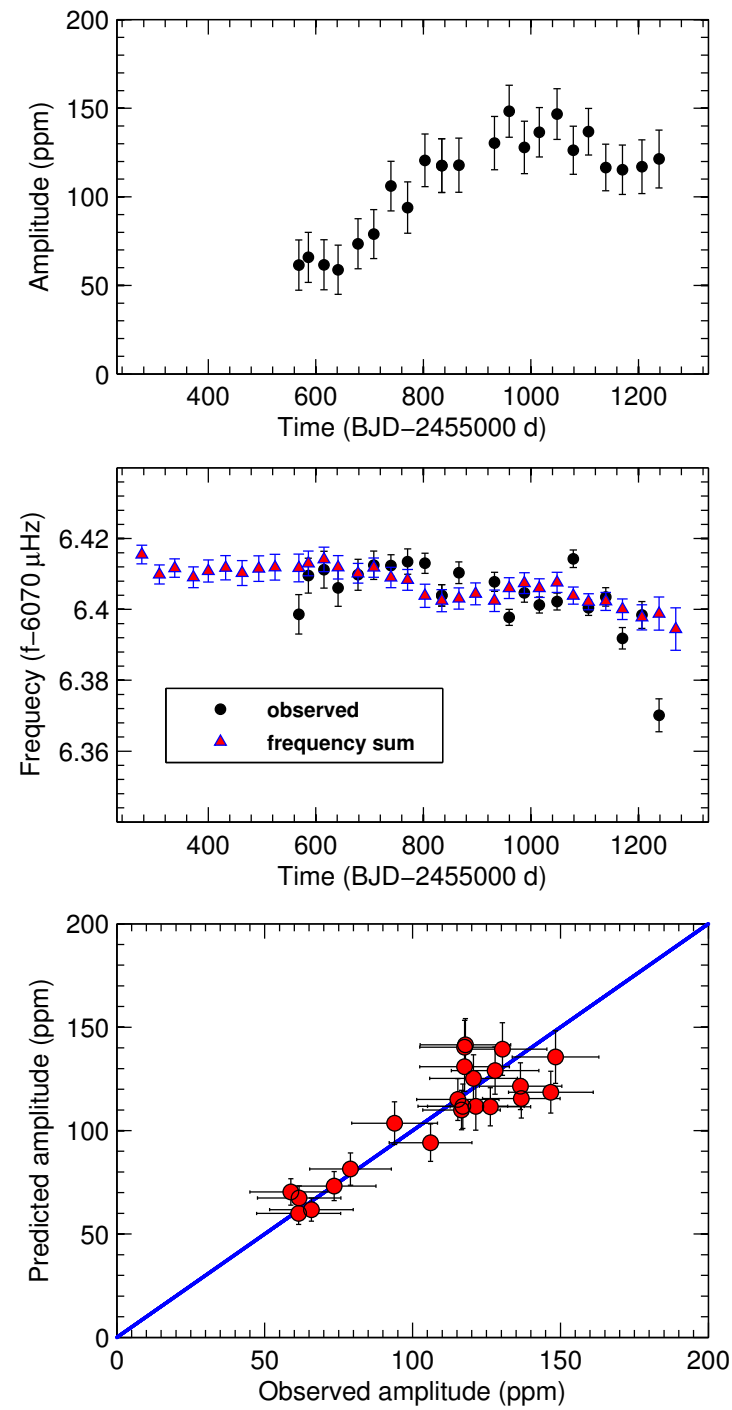

Fig. 14. Same as Fig. 13 but for the linear combination frequency $f_{35}=$ $f_{3}+f_{21}$. Note that there are ten missing measurements for $f_{21}$, including the first nine data points, because the amplitudes were lower than $4 \sigma$. The last data point is also not shown because of a large associated error, this measurement being at the end of the data set.

which involves one overstable parent mode and two damped unstable daughter modes.

At this stage, it becomes natural to interpret the complex variations observed in the components of $T_{1}$ and $T_{2}$ to be linked with the fact that these modes are simultaneously involved in two different types of resonances, that is, a triplet resonance and $v_{0} \sim v_{1}+v_{2}$ direct resonances. In this situation, the triplet resonance may be the dominating nonlinear interaction occurring in the triplet, while the nonlinear coupling with the modes outside the triplet could strongly perturb the periodic amplitude and frequency modulations expected if the triplets were pure isolated systems. This shows that nonlinear mode interactions in real stars are certainly more complex configurations than those treated by current simplified theoretical approaches. Moreover, since the $T_{2}$ triplet is in the transitory regime, with frequencies locked by the nonlinear coupling within the triplet, the nonlinear interactions outside this triplet are therefore unable to destroy this frequency locking, resulting in no long-term frequency variation as can be seen in the $T_{1}$ triplet. Interestingly, the resonant mode coupling theory predicts that a limit cycle (steady equilibrium state) may not be reached in the case of three-mode direct 
resonance (which is likely at work here as discussed above). The evolution of this long-term frequency variation in $T_{1}$, whether the mode frequencies will further converge or eventually diverge, and the evolution of $T_{2}$, whether frequencies will remain constant or the locked regime will eventually be broken, will need further observation either from ground or by future space instruments currently in preparation such as TESS and PLATO (Rauer et al. 2014; Ricker et al. 2014).

\subsection{The D-parameter and further insight on the modulations}

The value of the $D$-parameter, that defines how far the modes are from the resonance center, is usually connected to the kind of regime a multiplet is in when undergoing resonant mode interactions. This $D$-value is in particular sensitive to the linear growth rate of the oscillation modes (see again Sect. 3).

For the frequency-locked regime that is observed in KIC 10139564 with the $T_{3}$ triplet, $D$ is near or exactly zero, as predicted by the AEs (Buchler et al. 1995; Goupil et al. 1998). The $D$-value for the other triplets $T_{1}\left(\delta v_{\mathrm{o}} \sim 0.0026 \mu \mathrm{Hz}\right)$ and $T_{2}\left(\delta v_{\mathrm{o}} \sim 0.0036 \mu \mathrm{Hz}\right)$ may reflects more their linear growth rates since, with very similar frequency mismatches, the two triplets are found to be in different regimes. The growth rate values are indeed substantially different between $p$ - and $g$-modes (Charpinet 1999; Fontaine et al. 2003). Assuming the growth rate for $p$-modes is of the order of $10^{-6} \mathrm{~s}^{-1}$ (Charpinet 1999), the corresponding $D$ value for the $T_{1}$ triplet would be far less than one, indicating that $T_{1}$ should be in the frequency-locked regime (Buchler et al. 1997; Goupil et al. 1998), but we find it to be in the intermediate regime. We note however that this estimate for the value of $D$ is based on the measured frequency mismatch which may not be representative of the unperturbed frequency asymmetry that enters in the definition of $D$. The latter is likely much larger (see Sect. 4.1), leading to a somewhat larger $D$-value more in line with the observed regime of the resonance for $T_{1}$. The $D$-value for the $g$-mode triplet $T_{2}$, for its part, could be much larger than that of $T_{1}$, considering the much smaller growth rates of the $\ell=1 \mathrm{~g}$-modes (Fontaine et al. 2003). Extended ranges for $D$ were also found in the DBV star KIC 08626021 (Z16), where the $D$-values for the triplets are at least two orders of magnitude larger than those suggested in Goupil et al. (1998). This suggests that the nonlinear behaviors not only depend on the magnitude of $D$, but also on the specific coupling coefficients for each specific mode (Buchler et al. 1995).

Further quantitative comparisons between the observed modulations and the theoretical framework would require to solve the amplitude equations for the specific case of KIC 10139564. This would require to calculate the coupling coefficients in the AEs, which, in principle, could be extracted from the observed amplitude and frequency modulations (Buchler et al. 1995). With these known coupling coefficients, one could then determine the ranges of $D$-values related to each different regime of the nonlinear resonance. A measurement of the growth rates of the oscillation modes would then possibly follow with the determination of this parameter, which may lead for the first time to an independent estimation of the linear nonadiabatic growth rates of the modes and a direct test of nonadiabatic pulsation calculations in sdB stars.

\section{Summary and conclusion}

While studying the high-quality and long-duration photometric data provided by the Kepler spacecraft on the pulsating sdB star
KIC 10139564, we have identified different patterns in the frequency and amplitude modulations of the oscillation modes belonging to several rotationally split multiplets or linear combination frequencies. These modulations show signatures that can be associated to nonlinear resonant mode coupling mechanisms that could occur between the multiplet components themselves and with other modes under certain conditions, that is, satisfying a $v_{0} \sim v_{1}+v_{2}$ resonance relationship. This is the first time that such signatures are quite clearly identified in pulsating hot $B$ subdwarf stars, and the second case reported so far for a compact pulsator monitored with Kepler photometry (see Z16).

We first reanalyzed the 38-month of Kepler photometry obtained for KIC 10139564, leading to the detection of 60 independent frequencies above a secured detection threshold $(5.6 \sigma$; see Table A.1). Among these, 29 frequencies consist of three triplets, one doublet, one quintuplet and two incomplete multiplets with $\ell>2$ (see Table 1). Another three detected frequencies are linked to other frequencies through linear combinations. Five additional groups of frequencies are found in the region between 5400 and $6400 \mu \mathrm{Hz}$, which have very complicated structures. Finaly, we also find 14 independent frequencies and two frequencies satisfying linear combination relationships that could be real as their amplitudes are between $5 \sigma$ and $5.6 \sigma$ above the noise. In general, our well secured frequencies are in good agreement with the former analysis from Baran et al. (2012). In this paper, we particularly concentrated our study on six multiplets and three linear combination frequencies observed near $6076 \mu \mathrm{Hz}$

We found different types of mode behaviors occurring in the above mentioned frequencies. A "short timescale" quasiperiodic amplitude and frequency modulations along with a slow trend of the frequencies to convergence toward each other occur in the dominant $p$-mode triplet near $5760 \mu \mathrm{Hz}\left(T_{1}\right)$. The $\sim 570$-day quasi-periodic frequency modulation evolve in antiphase between the two side components in this triplet. Modulated frequencies and amplitudes are also found in a quintuplet near $5287 \mu \mathrm{Hz}\left(Q_{1}\right)$ and a $(\ell>2)$ multiplet near $5412 \mu \mathrm{Hz}$ $\left(M_{1}\right)$, but the modulations do not show a clear periodicity. One triplet near $316 \mu \mathrm{Hz}\left(T_{2}\right)$ has a distinct behavior from the above mentioned multiplets, as it shows stable frequencies but varying amplitudes. A similar phenomenon occurs in a doublet near $394 \mu \mathrm{Hz}\left(D_{1}\right)$ which shows constant frequencies and an $\sim 1100$ days periodic amplitude modulations. Another triplet at $518 \mu \mathrm{Hz}\left(T_{3}\right)$ completely differs from all the above multiplets, with constant amplitudes and frequencies throughout the whole observation run. In addition, we also discovered amplitude and frequency variations in three frequencies near $6076 \mu \mathrm{Hz}\left(C_{1}\right)$ that are linked to other independent frequencies through linear combinations.

After ruling out various possible causes for the modulations, we showed that these mode behaviors could be related to the different types of nonlinear resonances that should occur according to the amplitude equation formalism. In particular, nonlinear resonant couplings within a multiplet can lead to three main regimes, all of which are possibly occurring in KIC 10139564. The multiplets $T_{1}, Q_{1}$ and $M_{1}$ can be associated with the intermediate regime of the resonance where the involved modes have modulated amplitudes and frequencies. The triplet $T_{2}$ and doublet $D_{1}$ have a different behavior that could be associated to a narrow transitory regime in which the frequencies of the modes can be locked (constant) while the amplitudes experience modulations. The behavior of the triplet $T_{3}$ is the unique case found in this star that can be associated to the frequency lock regime of the resonance, where both amplitudes and frequencies are stable. In addition, the large amplitude ratios between the $C_{1}$ 
frequencies and their main parent modes, together with the large variation of amplitude and frequency observed for these peaks, suggest that $C_{1}$ correspond to three-mode direct resonances. We indeed found that the frequencies of $C_{1}$ exactly follow the evolution of their main parent modes. Moreover, as the parent modes of $C_{1}$ are also the components of $T_{1}$ and $T_{2}$, we suggest part of the complexity of the mode behaviors could be related to these cross interactions between the various modes. In particular, the slow variations occurring in $T_{1}$ may be related to the $v_{0} \sim v_{1}+v_{2}$ resonance superimposed to the triplet resonance occurring between the components.

We emphasize that the observed frequency modulations likely induced by nonlinear mode interactions could challenge future attempts to measure the evolutionary effects on the oscillation mode periods in pulsating sdB stars. Compared to the resonant variations taking place on timescales of years, the rate of period change of the pulsations due to stellar evolution in $\mathrm{sdB}$ stars is much longer, typically occurring on a timescale of $\sim 10^{6} \mathrm{yr}$ (Charpinet et al. 2002). Nonlinear modulations of the frequencies can potentially jeopardize any attempt to measure reliably such rates, unless they can be corrected beforehand. These nonlinear modulations could also complicate the detection of exoplanets or stellar companions around sdB stars using the technique of measuring phase changes in the pulsations (Silvotti et al. 2007). It should be possible however to distinguish between the two effects, considering that nonlinear couplings may induce different behaviors on different modes, while external causes such as an orbiting body should affect all modes similarly.

Finally, we note that our analysis suggests that resonances occurring in real stars, in which modes could be involved in two or more types of different couplings, lead to more complicated patterns than those predicted by current theoretical frameworks which treat the modes only as isolated systems within one type of resonance and ignore the nonlinear interactions that could occur simultaneously outside of the system. This should motivate further theoretical work to develop nonlinear stellar pulsation theory for more precise predictions of the mode behaviors in pulsating stars in general.

Acknowledgements. Funding for the Kepler mission is provided by NASA's Science Mission Directorate. We greatfully acknowledge the Kepler Science Team and all those who have contributed to making the Kepler mission possible. WKZ acknowledges the financial support from the China Scholarship Council. This work was supported in part by the Programme National de Physique Stellaire (PNPS, CNRS/INSU, France) and the Centre National d'Études Spatiales (CNES, France).

\section{References}

Baran A. S., \& Østensen R. H. 2013, Acta Astron., 63, 79 Baran, A., Pigulski, A., O’Toole, S. J. 2008, MNRAS, 385, 255
Baran A. S. Reed, M. D., Stello, D., et al. 2012, MNRAS, 424, 2686 Brassard, P., Fontaine, G., \& Wesemael, F. 1995, ApJS, 96, 545 Breger, M., \& Montgomery, M. H. 2014, ApJ, 783, 89 Buchler, J. R., \& Goupil, M. J. 1984, ApJ, 279, 384

Buchler, J. R., \& Kovacs, G. 1986, ApJ, 303, 794

Buchler, J. R., Goupil, M.-J., \& Serre, T. 1995, A\&A, 296, 405 Buchler, J. R., Goupil, M.-J., \& Hansen, C. J. 1997, A\&A, 321, 159 Carroll, B. W., \& Hansen, C. J. 1982, ApJ, 279, 394

Charpinet, S. 1999, Ph.D. Thesis, Univ. Montréal

Charpinet, S., Fontaine, G., Brassard, P., \& Dorman, B. 1996, ApJ, 471, L103

Charpinet, S., Fontaine, G., Brassard, P., et al. 1997, ApJ, 483, L123

Charpinet, S., Fontaine, G., Brassard, P., \& Dorman, B. 2002, ApJS, 140, 469

Charpinet, S., Green, E. M., Baglin, A., et al. 2010, A\&A, 516, L6

Charpinet, S., Van Grootel, V., Fontaine, G., et al. 2011, A\&A, 530, A3

Deeming, T. J. 1975, Ap\&SS, 36, 137

Dziembowski, W. A. 1982, Acta Astron., 32, 147

Dziembowski, W. A., \& Goode, P. R. 1992, ApJ, 394, 670

Fontaine, G., Brassard, P., Charpinet, S., et al. 2003, ApJ, 597, 518

Fontaine, G., Brassard, P., Charpinet, S., et al. 2012, A\&A, 539, A12

Gilliland, R. L., Timothy, M., Christensen-Dalsgaard, J., et al. 2010, PASP, 122, 131

Green, E. M., Fontaine, G., Reed, M. D., et al. 2003, ApJ, 583, L31

Goupil, M. J., \& Buchler, J. R. 1994, A\&A, 291, 484

Goupil, M. J., Dziembowski, W. A., \& Fontaine, G. 1998, Balt. Astron., 7, 21

Heber, U. 2009, ARA\&A, 47, 211

Jenkins, J. M., Caldwell, D. A., Chandrasekaran, H., et al. 2010, ApJ, 713, L87

Jones, P. W., Hansen, C. J., Pesnell, W. D., \& Kawaler, S. D. 1989, ApJ, 336, 403

Kawaler, S. D., Reed, M. D., Quint, A. C., et al. 2010, MNRAS, 409, 1487

Kilkenny, D. 2007, Commun. Asteroseismol., 150, 234

Kilkenny, D. 2010, Ap\&SS, 329, 175

Kilkenny, D., Koen, C., O’Donoghue, D., \& Stobie, R. S. 1997, MNRAS, 285, 640

Landstreet, J. D., Bagnulo, S., Fossati, L., Jordan, S., \& O’Toole, S. J. 2012, A\&A, 541, A100

Montgomery, M. H., \& Odonoghue, D. 1999, Delta Scuti Star Newsletter, 13, 28

Moskalik, P. 1985, Acta Astron., 35, 229

Østensen, R. H., Reed, M. D., Baran, A. S., \& Telting, J. H. 2014, A\&A, 564, L14

Petit, P., Van Grootel, V., Bagnulo, S., et al. 2012, in Fifth Meeting on Hot Subdwarf Stars and Related Objects, ASP Conf. Ser., 452, 87

Rauer, H., Catala, C., Aerts, C., et al. 2014, Exp. Astron., 38, 249

Reed, M. D., O’Toole, S. J., Terndrup, D. M., et al. 2007, ApJ, 664, 518

Ricker, G. R., Winn, J. N., Vanderspek, R., et al. 2014, SPIE, 9143E, 20

Saio, H. 1981, ApJ, 244, 299

Salabert, D., García, R. A., \& Turck-Chièze, S. 2015, A\&A, 578, A137

Scargle, J. D. 1982, ApJ, 263, 835

Schuh, S., Hubber, J., Dreizler, S., et al. 2006, A\&A, 445, 131

Silvotti, R., Schuh, S., Janulis, R., et al. 2007, Nature, 449, 189

Unno, W., Osaki, Y., Ando, H., Saio, H., \& Shibahashi, H. 1989, Nonradial oscillations of stars, 2nd edn. (Tokyo: University of Tokyo Press)

van Grootel, V., Charpinet, S., Fontaine, G., et al. 2010, ApJ, 718, L97

Van Hoolst, T. 1994, A\&A, 292, 183

van Kerkwijk, M. H., Clemens, J. C., \& Wu, Y. 2000, MNRAS, 314, 209

Vauclair, G., Fu, J.-N., Solheim, J. E., et al. 2011, A\&A, 528, A5

Wu, Y. 2001, MNRAS, 323, 248

Wu, Y., \& Goldreich, P. 2001, ApJ, 546, 469

Zong, W., Charpinet, S., Vauclair, G., et al. 2016, A\&A, 585, A22 (Z16) 


\section{Appendix A: Additional table}

Table A.1. List of frequencies detected in KIC 10139564.

\begin{tabular}{|c|c|c|c|c|c|c|c|c|c|c|}
\hline Id. & $\begin{array}{c}\text { Frequency } \\
(\mu \mathrm{Hz})\end{array}$ & $\begin{array}{c}\sigma_{\mathrm{f}} \\
(\mu \mathrm{Hz})\end{array}$ & $\begin{array}{c}\text { Period } \\
(\mathrm{s})\end{array}$ & $\begin{array}{l}\sigma_{\mathrm{P}} \\
(\mathrm{s})\end{array}$ & $\begin{array}{c}\text { Amplitude } \\
(\%)\end{array}$ & $\begin{array}{l}\sigma_{\mathrm{A}} \\
(\%)\end{array}$ & Phase & $\sigma_{\mathrm{Ph}}$ & $S / N$ & ${ }^{\dagger}$ Comment \\
\hline \multicolumn{11}{|c|}{ Multiplet frequencies: } \\
\hline$f_{39}$ & 315.579243 & 0.000566 & 3168.776214 & 0.005687 & 0.005851 & 0.000596 & 0.2492 & 0.0516 & 9.8 & $T_{2,-1}$ \\
\hline$f_{21}$ & 315.820996 & 0.000219 & 3166.350599 & 0.002193 & 0.015155 & 0.000596 & 0.6107 & 0.0199 & 25.4 & $T_{2,0}$ \\
\hline$f_{11}$ & 316.066440 & 0.000070 & 3163.891744 & 0.000702 & 0.047276 & 0.000596 & 0.2063 & 0.0064 & 79.3 & $T_{2,+1}$ \\
\hline$f_{27}$ & 394.027385 & 0.000342 & 2537.894669 & 0.002202 & 0.009667 & 0.000594 & 0.2589 & 0.0312 & 16.3 & $D_{1,0}$ \\
\hline$f_{32}$ & 394.289823 & 0.000397 & 2536.205455 & 0.002555 & 0.008323 & 0.000594 & 0.5123 & 0.0363 & 14.0 & $D_{1,+1}$ \\
\hline$f_{34}$ & 518.900359 & 0.000437 & 1927.152262 & 0.001624 & 0.007526 & 0.000592 & 0.6648 & 0.0401 & 12.7 & $T_{3,-1}$ \\
\hline$f_{28}$ & 519.151796 & 0.000352 & 1926.218898 & 0.001305 & 0.009351 & 0.000592 & 0.9059 & 0.0323 & 15.8 & $T_{3,0}$ \\
\hline$f_{31}$ & 519.402391 & 0.000367 & 1925.289559 & 0.001360 & 0.008964 & 0.000592 & 0.5369 & 0.0337 & 15.2 & $T_{3,+1}$ \\
\hline$f_{08}$ & 5286.149823 & 0.000053 & 189.173601 & 0.000002 & 0.064784 & 0.000614 & 0.6712 & 0.0047 & 105.4 & $Q_{1,-2}$ \\
\hline$f_{10}$ & 5286.561766 & 0.000060 & 189.158861 & 0.000002 & 0.057105 & 0.000614 & 0.4356 & 0.0053 & 92.9 & $Q_{1,-1}$ \\
\hline$f_{07}$ & 5286.976232 & 0.000038 & 189.144032 & 0.000001 & 0.088857 & 0.000614 & 0.1202 & 0.0034 & 144.6 & $Q_{1,0}$ \\
\hline$f_{05}$ & 5287.391879 & 0.000019 & 189.129163 & 0.000001 & 0.179339 & 0.000615 & 0.3374 & 0.0017 & 291.8 & $Q_{1,+1}$ \\
\hline$f_{06}$ & 5287.805883 & 0.000029 & 189.114355 & 0.000001 & 0.119329 & 0.000615 & 0.7941 & 0.0025 & 194.2 & $Q_{1,+2}$ \\
\hline$f_{22}$ & 5410.701146 & 0.000234 & 184.818931 & 0.000008 & 0.014871 & 0.000627 & 0.9524 & 0.0203 & 23.7 & $M_{1,0}$ \\
\hline$f_{67}$ & 5411.143448 & 0.000958 & 184.803824 & 0.000033 & 0.003637 & 0.000627 & 0.4591 & 0.0830 & 5.8 & $M_{1,0}$ \\
\hline$f_{13}$ & 5411.597301 & 0.000136 & 184.788325 & 0.000005 & 0.025636 & 0.000627 & 0.6770 & 0.0118 & 40.9 & $M_{1,0}$ \\
\hline$f_{15}$ & 5412.516444 & 0.000185 & 184.756944 & 0.000006 & 0.018812 & 0.000627 & 0.8925 & 0.0160 & 30.0 & $M_{1,0}$ \\
\hline$f_{12}$ & 5413.389096 & 0.000084 & 184.727161 & 0.000003 & 0.041339 & 0.000627 & 0.4037 & 0.0073 & 65.9 & $M_{1,0}$ \\
\hline$f_{19}$ & 5413.814342 & 0.000222 & 184.712651 & 0.000008 & 0.015718 & 0.000627 & 0.7225 & 0.0192 & 25.1 & $M_{1,0}$ \\
\hline$f_{25}$ & 5570.030091 & 0.000389 & 179.532244 & 0.000013 & 0.010056 & 0.000703 & 0.5938 & 0.0300 & 14.3 & $M_{2,0}$ \\
\hline$f_{56}$ & 5570.484768 & 0.000964 & 179.517590 & 0.000031 & 0.004058 & 0.000703 & 0.8087 & 0.0744 & 5.8 & $M_{2,0}$ \\
\hline$f_{61}$ & 5570.937140 & 0.001001 & 179.503013 & 0.000032 & 0.003913 & 0.000704 & 0.8254 & 0.0772 & 5.6 & $M_{2,0}$ \\
\hline$f_{29}$ & 5571.393930 & 0.000421 & 179.488295 & 0.000014 & 0.009297 & 0.000705 & 0.5332 & 0.0325 & 13.2 & $M_{2,0}$ \\
\hline$f_{43}$ & 5572.293674 & 0.000760 & 179.459314 & 0.000024 & 0.005168 & 0.000706 & 0.5854 & 0.0584 & 7.3 & $M_{2,0}$ \\
\hline$f_{50}$ & 5572.728096 & 0.000902 & 179.445324 & 0.000029 & 0.004356 & 0.000707 & 0.5037 & 0.0693 & 6.2 & $M_{2,0}$ \\
\hline$f_{45}$ & 5708.908076 & 0.000897 & 175.164845 & 0.000028 & 0.004648 & 0.000749 & 0.0571 & 0.0650 & 6.2 & $M_{2,0}$ \\
\hline$f_{01}$ & 5760.167840 & 0.000005 & 173.606052 & & 0.825132 & 0.000761 & 0.0744 & 0.0004 & 1084.9 & $T_{1,-1}$ \\
\hline$f_{03}$ & 5760.586965 & 0.000008 & 173.593421 & & 0.554646 & 0.000761 & 0.6388 & 0.0005 & 729.3 & $T_{1,0}$ \\
\hline$f_{02}$ & 5761.008652 & 0.000007 & 173.580715 & & 0.567034 & 0.000761 & 0.5845 & 0.0005 & 745.5 & $T_{1,+1}$ \\
\hline \multicolumn{11}{|c|}{ Independent frequencies: } \\
\hline$f_{72}$ & 892.042910 & 0.000986 & 1121.022305 & 0.001239 & 0.003319 & 0.000588 & 0.6058 & 0.0909 & 5.6 & \\
\hline$f_{70}$ & 2212.606534 & 0.000942 & 451.955639 & 0.000192 & 0.003462 & 0.000586 & 0.0639 & 0.0872 & 5.9 & \\
\hline$f_{37}$ & 3540.459896 & 0.000514 & 282.449182 & 0.000041 & 0.006309 & 0.000583 & 0.3837 & 0.0478 & 10.8 & \\
\hline$f_{36}$ & 3541.431179 & 0.000477 & 282.371716 & 0.000038 & 0.006796 & 0.000583 & 0.4013 & 0.0444 & 11.7 & \\
\hline$f_{47}$ & 4064.355754 & 0.000719 & 246.041454 & 0.000044 & 0.004526 & 0.000585 & 0.4296 & 0.0667 & 7.7 & \\
\hline$f_{46}$ & 5048.744283 & 0.000729 & 198.069053 & 0.000029 & 0.004548 & 0.000596 & 0.9108 & 0.0664 & 7.6 & \\
\hline$f_{41}$ & 5049.709963 & 0.000610 & 198.031176 & 0.000024 & 0.005432 & 0.000596 & 0.6548 & 0.0556 & 9.1 & \\
\hline$f_{63}$ & 5052.604965 & 0.000879 & 197.917709 & 0.000034 & 0.003771 & 0.000596 & 0.5451 & 0.0800 & 6.3 & \\
\hline$f_{04}$ & 5472.861431 & 0.000007 & 182.719773 & & 0.476915 & 0.000638 & 0.2824 & 0.0006 & 747.9 & \\
\hline$f_{42}$ & 5709.026672 & 0.000793 & 175.161207 & 0.000024 & 0.005254 & 0.000749 & 0.0777 & 0.0575 & 7.0 & \\
\hline$f_{53}$ & 5740.666960 & 0.001002 & 174.195787 & 0.000030 & 0.004168 & 0.000751 & 0.8817 & 0.0724 & 5.6 & \\
\hline$f_{49}$ & 5740.807435 & 0.000946 & 174.191525 & 0.000029 & 0.004411 & 0.000751 & 0.0263 & 0.0684 & 5.9 & \\
\hline$f_{50}$ & 5746.615392 & 0.000612 & 174.015474 & 0.000019 & 0.006881 & 0.000757 & 0.0989 & 0.0439 & 9.1 & \\
\hline$f_{18}$ & 5747.099099 & 0.000261 & 174.000828 & 0.000008 & 0.016157 & 0.000757 & 0.8738 & 0.0187 & 21.3 & \\
\hline$f_{17}$ & 5748.065581 & 0.000257 & 173.971571 & 0.000008 & 0.016414 & 0.000758 & 0.5427 & 0.0184 & 21.7 & \\
\hline$f_{16}$ & 5749.067189 & 0.000256 & 173.941262 & 0.000008 & 0.016476 & 0.000758 & 0.1319 & 0.0183 & 21.7 & \\
\hline
\end{tabular}

Notes. ${ }^{(\dagger)}$ The first subscript is the identity of the multiplet and the second one indicates the value of $m$. The $m$-values for two $\ell>2$ multiplets, $M_{1}$ and $M_{2}$, are not provided as the degree $\ell$ is not known. 
W. Zong et al.: Signatures of nonlinear mode interactions in the pulsating hot B subdwarf star KIC 10139564

Table A.1. continued.

\begin{tabular}{|c|c|c|c|c|c|c|c|c|c|c|}
\hline Id. & $\begin{array}{c}\text { Frequency } \\
(\mu \mathrm{Hz})\end{array}$ & $\begin{array}{c}\sigma_{\mathrm{f}} \\
(\mu \mathrm{Hz})\end{array}$ & $\begin{array}{l}\text { Period } \\
\text { (s) }\end{array}$ & $\begin{array}{l}\sigma_{\mathrm{P}} \\
(\mathrm{s})\end{array}$ & $\begin{array}{c}\text { Amplitude } \\
(\%)\end{array}$ & $\begin{array}{l}\sigma_{\mathrm{A}} \\
(\%)\end{array}$ & Phase & $\sigma_{\mathrm{Ph}}$ & $S / N$ & ${ }^{\dagger}$ Comment \\
\hline$f_{44}$ & 5840.820662 & 0.000903 & 171.208818 & 0.000026 & 0.004685 & 0.000761 & 0.7872 & 0.0645 & 6.2 & \\
\hline$f_{65}$ & 6057.645946 & 0.000970 & 165.080629 & 0.000026 & 0.003740 & 0.000652 & 0.2785 & 0.0815 & 5.7 & \\
\hline$f_{55}$ & 6057.688799 & 0.000876 & 165.079461 & 0.000024 & 0.004142 & 0.000652 & 0.9775 & 0.0736 & 6.3 & \\
\hline$f_{66}$ & 6106.662077 & 0.000977 & 163.755582 & 0.000026 & 0.003675 & 0.000646 & 0.6890 & 0.0822 & 5.7 & \\
\hline$f_{60}$ & 6757.710494 & 0.000838 & 147.979112 & 0.000018 & 0.003938 & 0.000594 & 0.1752 & 0.0767 & 6.6 & \\
\hline$f_{59}$ & 6758.215141 & 0.000835 & 147.968062 & 0.000018 & 0.003954 & 0.000594 & 0.1869 & 0.0764 & 6.7 & \\
\hline$f_{30}$ & 7633.720521 & 0.000360 & 130.997722 & 0.000006 & 0.009090 & 0.000589 & 0.1759 & 0.0332 & 15.4 & \\
\hline$f_{40}$ & 7634.190048 & 0.000592 & 130.989665 & 0.000010 & 0.005536 & 0.000589 & 0.3321 & 0.0546 & 9.4 & \\
\hline$f_{64}$ & 7634.677476 & 0.000873 & 130.981302 & 0.000015 & 0.003753 & 0.000589 & 0.1106 & 0.0805 & 6.4 & \\
\hline$f_{52}$ & 8118.752590 & 0.000768 & 123.171631 & 0.000012 & 0.004284 & 0.000591 & 0.3497 & 0.0705 & 7.2 & \\
\hline$f_{33}$ & 8119.248304 & 0.000436 & 123.164111 & 0.000007 & 0.007538 & 0.000591 & 0.4515 & 0.0400 & 12.7 & \\
\hline$f_{48}$ & 8496.107048 & 0.000733 & 117.700965 & 0.000010 & 0.004503 & 0.000594 & 0.8648 & 0.0744 & 7.6 & \\
\hline$f_{71}$ & 8496.293646 & 0.000973 & 117.698380 & 0.000013 & 0.003395 & 0.000594 & 0.0896 & 0.0934 & 5.7 & \\
\hline$f_{54}$ & 8615.236287 & 0.000795 & 116.073427 & 0.000011 & 0.004159 & 0.000594 & 0.5932 & 0.0727 & 7.0 & \\
\hline$f_{38}$ & 8616.169582 & 0.000539 & 116.060854 & 0.000007 & 0.006128 & 0.000594 & 0.7833 & 0.0498 & 10.3 & \\
\hline \multicolumn{11}{|c|}{ Linear combination frequencies: } \\
\hline$f_{23}$ & 6076.234996 & 0.000252 & 164.575597 & 0.000007 & 0.014360 & 0.000650 & 0.7906 & 0.0210 & 22.1 & $f_{11}+f_{01}$ \\
\hline$f_{35}$ & 6076.408232 & 0.000510 & 164.570905 & 0.000014 & 0.007091 & 0.000650 & 0.7821 & 0.0426 & 10.9 & $f_{21}+f_{03}$ \\
\hline$f_{74}$ & 6076.650684 & 0.001120 & 164.564338 & 0.000030 & 0.003225 & 0.000650 & 0.5520 & 0.0937 & 5.0 & $f_{11}+f_{03}$ \\
\hline$f_{68}$ & 190.138219 & 0.000959 & 5259.331906 & 0.026527 & 0.003563 & 0.000614 & 0.2639 & 0.0847 & 5.8 & $f_{01}-f_{25}$ \\
\hline$f_{79}$ & 287.306296 & 0.001081 & 3480.605932 & 0.013093 & 0.003075 & 0.000598 & 0.9190 & 0.0982 & 5.1 & $f_{01}-f_{04}$ \\
\hline \multicolumn{11}{|c|}{ Group frequencies: } \\
\hline$f_{20}$ & 5471.730865 & 0.000230 & 182.757527 & 0.000008 & 0.015410 & 0.000637 & 0.9397 & 0.0196 & 24.2 & G1 \\
\hline$f_{14}$ & 5944.170986 & 0.000209 & 168.232038 & 0.000006 & 0.019127 & 0.000720 & 0.1222 & 0.0158 & 26.6 & $\mathrm{G} 2$ \\
\hline$f_{24}$ & 6001.472409 & 0.000273 & 166.625776 & 0.000008 & 0.013532 & 0.000664 & 0.2224 & 0.0223 & 20.4 & G3 \\
\hline$f_{26}$ & 6172.852132 & 0.000353 & 161.999669 & 0.000009 & 0.009980 & 0.000633 & 0.6961 & 0.0302 & 15.8 & G4 \\
\hline$f_{09}$ & 6234.713029 & 0.000058 & 160.392306 & 0.000001 & 0.062028 & 0.000648 & 0.9590 & 0.0072 & 95.7 & G5 \\
\hline$f_{51}$ & 6315.214679 & 0.000798 & 158.347744 & 0.000020 & 0.004312 & 0.000619 & 0.3510 & 0.0700 & 7.0 & G6 \\
\hline \multicolumn{11}{|c|}{ Suspected frequencies: } \\
\hline$f_{77}$ & 4061.893709 & 0.001051 & 246.190588 & 0.000064 & 0.003097 & 0.000585 & 0.9507 & 0.0974 & 5.3 & \\
\hline$f_{57}$ & 5838.962703 & 0.001063 & 171.263296 & 0.000031 & 0.003980 & 0.000761 & 0.6543 & 0.0758 & 5.2 & \\
\hline$f_{62}$ & 5841.187712 & 0.001114 & 171.198059 & 0.000033 & 0.003796 & 0.000761 & 0.7222 & 0.0796 & 5.0 & \\
\hline$f_{58}$ & 5841.581605 & 0.001068 & 171.186516 & 0.000031 & 0.003960 & 0.000761 & 0.1969 & 0.0762 & 5.2 & \\
\hline$f_{83}$ & 345.231189 & 0.001151 & 2896.609673 & 0.009656 & 0.002878 & 0.000596 & 0.8214 & 0.1050 & 4.8 & \\
\hline$f_{84}$ & 345.597339 & 0.001193 & 2893.540798 & 0.009988 & 0.002777 & 0.000596 & 0.3501 & 0.1088 & 4.7 & \\
\hline$f_{78}$ & 345.976695 & 0.001077 & 2890.368090 & 0.008999 & 0.003076 & 0.000596 & 0.4839 & 0.0982 & 5.2 & \\
\hline$f_{69}$ & 6106.245918 & 0.001014 & 163.766742 & 0.000027 & 0.003544 & 0.000646 & 0.6171 & 0.0852 & 5.5 & \\
\hline$f_{75}$ & 6418.164502 & 0.001068 & 155.807786 & 0.000026 & 0.003178 & 0.000610 & 0.7474 & 0.0950 & 5.2 & \\
\hline$f_{76}$ & 6758.687089 & 0.001049 & 147.957730 & 0.000023 & 0.003148 & 0.000594 & 0.5124 & 0.0959 & 5.3 & \\
\hline$f_{80}$ & 6997.352981 & 0.001084 & 142.911184 & 0.000022 & 0.003003 & 0.000586 & 0.5103 & 0.1005 & 5.1 & \\
\hline$f_{73}$ & 7633.957044 & 0.001007 & 130.993663 & 0.000017 & 0.003251 & 0.000589 & 0.2483 & 0.0930 & 5.5 & \\
\hline$f_{81}$ & 8117.287298 & 0.001114 & 123.193866 & 0.000017 & 0.002953 & 0.000591 & 0.7915 & 0.1022 & 5.0 & \\
\hline$f_{82}$ & 8377.175646 & 0.001119 & 119.371975 & 0.000016 & 0.002937 & 0.000591 & 0.8589 & 0.1040 & 5.0 & \\
\hline
\end{tabular}

